\title{
Triaxially deformed relativistic point-coupling model for $\Lambda$ hypernuclei: a quantitative analysis of hyperon impurity effect on nuclear collective properties
}

\author{
W. X. Xue, ${ }^{1}$ J. M. Yao, ${ }^{1,2, *}$ K. Hagino,${ }^{2,3}$ Z. P. Li, ${ }^{1}$ H. Mei, ${ }^{2,1}$ and Y. Tanimura ${ }^{4}$ \\ ${ }^{1}$ School of Physical Science and Technology, Southwest University, Chongqing 400715, China \\ ${ }^{2}$ Department of Physics, Tohoku University, Sendai 980-8578, Japan \\ ${ }^{3}$ Research Center for Electron Photon Science, Tohoku University, 1-2-1 Mikamine, Sendai 982-0826, Japan \\ ${ }^{4}$ Institut de Physique Nucléaire, IN2P3-CNRS, Université Paris-Sud, F-91406 Orsay Cedex, France
}

Background The impurity effect of hyperon on atomic nuclei has received a renewed interest in nuclear physics since the first experimental observation of appreciable reduction of $E 2$ transition strength in low-lying states of hypernucleus ${ }_{\Lambda}^{7} \mathrm{Li}$. Many more data on low-lying states of $\Lambda$ hypernuclei will be measured soon for $s d$-shell nuclei, providing good opportunities to study the $\Lambda$ impurity effect on nuclear low-energy excitations.

Purpose We carry out a quantitative analysis of $\Lambda$ hyperon impurity effect on the low-lying states of $s d$-shell nuclei at the beyond-mean-field level based on a relativistic point-coupling energy density functional (EDF), considering that the $\Lambda$ hyperon is injected into the lowest positive-parity $\left(\Lambda_{s}\right)$ and negative-parity $\left(\Lambda_{p}\right)$ states.

Method We adopt a triaxially deformed relativistic mean-field (RMF) approach for hypernuclei and calculate the $\Lambda$ binding energies of hypernuclei as well as the potential energy surfaces (PESs) in $(\beta, \gamma)$ deformation plane. We also calculate the PESs for the $\Lambda$ hypernuclei with good quantum numbers using a microscopic particle rotor model (PRM) with the same relativistic EDF. The triaxially deformed RMF approach is further applied in order to determine the parameters of a five-dimensional collective Hamiltonian $(5 \mathrm{DCH})$ for the collective excitations of triaxially deformed core nuclei. Taking ${ }_{\Lambda}^{25,27} \mathrm{Mg}$ and ${ }_{\Lambda}^{31} \mathrm{Si}$ as examples, we analyse the impurity effects of $\Lambda_{s}$ and $\Lambda_{p}$ on the low-lying states of the core nuclei.

Results We show that $\Lambda_{s}$ increases the excitation energy of the $2_{1}^{+}$state and decreases the $E 2$ transition strength from this state to the ground state by $12 \%-17 \%$. On the other hand, $\Lambda_{p}$ tends to develop pronounced energy minima with larger deformation, although it modifies the collective parameters in such a way that the collectivity of the core nucleus can be either increased or decreased.

Conclusions The quadrupole deformation significantly affects the $\Lambda$ binding energies of deformed hypernuclei. A beyondmean-field approach with the dynamical correlations due to restoration of broken symmetries and shape fluctuation is essential in order to study the $\Lambda$ impurity effect in a quantitative way.

PACS numbers: 21.80.+a, 21.60.Jz, 21.60.Ev

\section{INTRODUCTION}

Since the first discovery of $\Lambda$ hypernuclei in $1953[1,2]$, hypernuclear physics as an important branch of nuclear physics has attracted lots of attention and many novel phenomena have been discovered in this field. Due to the absence of Pauli exclusion principle from other nucleons, a hyperon with strangeness degree-of-freedom can probe deeply into the interior of nuclear medium and significantly modify nuclear properties. For example, a hyperon may induce a softening of the equation of state of nuclear matter changing the properties of neutron stars [3], a shrinkage of the size of atomic nuclei with cluster structure [4-6], a stabilization of the binding of unbound nuclear systems [7] and thus the driplines of nucleons [8], a modification of nuclear deformation [9, 10] and collective excitations [11-14], and a reduction of fissionbarrier height in heavy nuclei $[15,16]$. Because hyperonnucleon and hyperon-hyperon scattering experiments are difficult to perform, the study of properties of hypernuclei has in fact been playing a vital role in understanding baryon-baryon interactions in nuclear medium, which are

\footnotetext{
*Electronic address: jmyao@swu.edu.cn
}

important not only for understanding hypernuclear structure but also for the study of hypernuclear matter and neutron stars [17]. A comprehensive introduction to the history and/or recent developments on various aspects in hypernuclear physics can be found in the review papers [18-25].

Thanks to the advent of hyperball facility for measuring hypernuclear $\gamma$-ray spectroscopy with high resolution $[6,26]$, the study of $\Lambda$ hyperon impurity effect on nuclear deformation and low-energy structure has attracted a renewed interest. The self-consistent mean-field (SCMF) approaches make good tools for this study because they provide a vivid way to investigate how the ground-state deformation is affected by adding a $\Lambda$ particle. In the past decades, the SCMF approaches have been adopted extensively to study the structure of hypernuclei [8, 27-38]. However, most of these studies are restricted to spherical systems. In recent years, the SCMF approaches have been extended to deformed cases in order to examine the change of nuclear deformation after adding a hyperon, based either on Skyrme forces [39-41] or on effective relativistic meson-exchange Lagrangian [9, 10, 42, 43]. It has been found in these mean-field studies that the deformations of hypernuclei and the corresponding core nucleus are rather similar, but with some exceptions as predicted by the relativistic 
mean-field (RMF) calculations $[9,10]$. It implies that the hyperon impurity effect is generally larger in the calculation with relativistic approaches than that with non-relativistic approaches, as has been pointed out by Schulze et al. [44]. Therefore, one would encounter more opportunities to see drastic deformation changes from ordinary nuclei to hypernuclei in the studies based on relativistic energy density functionals (EDFs).

It should be pointed out that, in most of the previous SCMF studies allowing deformation, the $\Lambda$ hyperon is put in the lowest positive-parity $\left(\Lambda_{s}\right)$ state. The corresponding $\Lambda_{s}$-hypernuclei turn out to have a softer energy surface than their core nucleus. This indicates that the dynamical shape fluctuation effect will be more important in hypernuclei than in normal nuclei, and thus the meanfield approaches might overestimate/underestimate the $\Lambda$ hyperon impurity on nuclear deformation and shapes. To quantify the $\Lambda$ hyperon impurity effect, one therefore has to go beyond-mean-field (BMF) approximation to take into account the dynamical correlation effects associated with symmetry restoration and shape fluctuation. Notice that the deformed mean-field breaks rotational symmetry, and, if one works only at the mean-field level, the connection of the deformed solution to spectroscopic observables, such as $B(E 2)$ value, has to rely on additional assumptions such as the rigid-rotor model, which becomes ill-defined in light and soft nuclei.

Recently, we have quantitatively studied the $\Lambda$ impurity effect on the low-lying states of ${ }^{24} \mathrm{Mg}$ by using a five-dimensional collective Hamiltonian (5DCH) as a choice of the BMF approaches [11]. The parameters of the $5 \mathrm{DCH}$ were determined by a triaxially deformed Skyrme-Hartree-Fock (SHF)+BCS calculation. We have found that the presence of one $\Lambda$ hyperon in the lowest positive-parity state reduces the $B\left(E 2: 2_{1}^{+} \rightarrow 0_{1}^{+}\right)$in ${ }^{24} \mathrm{Mg}$ by $9 \%$ and shifts up the excitation energy of the second $2^{+}$state by about $240 \mathrm{keV}$. Similar conclusions have also been found in the BMF study based on the antisymmetrized molecular dynamics (AMD) model $[12,45]$. We note that these BMF studies are within non-relativistic frameworks. Moreover, the impurity effect of $\Lambda$ hyperon in the lowest negative-parity $\left(\Lambda_{p}\right)$ state has not been well examined in the $5 \mathrm{DCH}$ approach.

In view of the above facts, it is interesting to quantitatively study the $\Lambda$ hyperon impurity effect based on a relativistic EDF at the BMF level by putting the $\Lambda$ hyperon in the $\Lambda_{s}$ and $\Lambda_{p}$ states. To this end, as a continuation of our previous work [11], we adopt the same 5DCH approach for the low-lying states of core nuclei but with collective parameters determined from a triaxial RMF + BCS calculation. We generalize our triaxial RMF approach in a three-dimensional harmonic-oscillator (3DHO) basis for ordinary nuclei [46] to $\Lambda$ hypernuclei by including $\Lambda$ hyperons. This 5DCH method based on the triaxial RMF solutions can be regarded as the Gaussian overlap approximation to the generator coordinate method (GCM) [47, 48] with three-dimensional angular momentum projection (3DAMP) [49]. The success of the $5 \mathrm{DCH}$ method based on relativistic EDFs has been illustrated in a series of calculations for spherical, transitional, and deformed nuclei from light to superheavy regions [50-54]. In particular, the validity of the $5 \mathrm{DCH}$ approach for the low-lying states of ${ }^{76} \mathrm{Kr}$ has recently been verified against a seven-dimensional GCM calculation [55].

It is worth mentioning that there are two other triaxially deformed RMF codes for $\Lambda$ hypernuclei based on meson-exchange interaction. One was developed by B. N. Lü et al. with an axially deformed HO basis [10, 42], while the other one was developed by H. F. Lü et al. [56] based on the triaxial RMF code with the $3 \mathrm{DHO}$ basis for ordinary nuclei [57] with time-odd fields but without pairing correlation. Our triaxial code developed in the present work includes the paring correlation and is mainly based on but not restricted to the relativistic point-coupling EDFs, which have been widely adopted to study nuclear low-lying states within the framework of multi-reference covariant density functional theory (CDFT) $[47,55,58,59]$.

The paper is arranged as follows. In Sec. II, we present the main formalism of the triaxial RMF approach for $\Lambda$ hypernuclei. In Sec. III, we present the results for $\Lambda$ binding energies obtained with the triaxial RMF code and compare to the results of the spherical code. In particular, we discuss the potential energy surfaces (PESs) for $\Lambda$ hypernuclei ${ }^{25,27} \mathrm{Mg},{ }_{\Lambda}^{31} \mathrm{Si}$ as well as the core nucleus of each of these hypernuclei in $(\beta, \gamma)$ deformation plane. In Sec. IV, the microscopic particle-rotor model (PRM) for the PES of $\Lambda$ hypernucleus ${ }_{\Lambda}^{25} \mathrm{Mg}$ with spin-parity of $I^{\pi}=1 / 2^{+}$and $1 / 2^{-}$are discussed in comparison with that of ${ }^{24} \mathrm{Mg}$ with $J^{\pi}=0^{+}$. In Sec. V, the $5 \mathrm{DCH}$ method is adopted to study a change in the low-lying states of the core nucleus by adding a $\Lambda$ hyperon. The impurity effect of $\Lambda$ hyperon is discussed both for $\Lambda_{s}$ and $\Lambda_{p}$. A summary of the present study and an outlook are then given in Sec. VI.

\section{TRIAXIALLY DEFORMED RELATIVISTIC MEAN-FIELD APPROACH FOR $\Lambda$ HYPERNUCLEI}

In the present triaxial RMF approach for $\Lambda$ hypernuclei, the nucleon-nucleon $(N N)$ and nucleon-hyperon $(N \Lambda)$ effective interactions are described in terms of contact couplings with different vertices. The Lagrangian density for $\Lambda$ hypernuclei then reads,

$$
\mathcal{L}=\mathcal{L}^{\text {free }}+\mathcal{L}^{\mathrm{em}}+\mathcal{L}^{N N}+\mathcal{L}^{N \Lambda}+\mathcal{L}^{\Lambda \Lambda}
$$

where the first term $\mathcal{L}^{\text {free }}$ is for the free nucleons and hyperon, and $\mathcal{L}^{\mathrm{em}}$ for the Coulomb interaction between protons. The third term $\mathcal{L}^{N N}$ is for the $N N$ effective interaction part. The detailed expressions for these terms can be found for example in Refs. [60, 61]. Since we focuse on single- $\Lambda$ hypernuclei in this work, the $\mathcal{L}^{\Lambda \Lambda}$ term 
for $\Lambda \Lambda$ interaction vanishes. The $N \Lambda$ interaction is chosen as in Ref. [62], that is,

$$
\mathcal{L}^{N \Lambda}=\mathcal{L}_{4 \mathrm{f}}^{N \Lambda}+\mathcal{L}_{\mathrm{der}}^{N \Lambda}+\mathcal{L}_{\mathrm{ten}}^{N \Lambda}
$$

with

$$
\begin{aligned}
\mathcal{L}_{4 \mathrm{f}}^{N \Lambda}= & -\alpha_{S}^{(N \Lambda)}\left(\bar{\psi}^{N} \psi^{N}\right)\left(\bar{\psi}^{\Lambda} \psi^{\Lambda}\right) \\
& -\alpha_{V}^{(N \Lambda)}\left(\bar{\psi}^{N} \gamma_{\mu} \psi^{N}\right)\left(\bar{\psi}^{\Lambda} \gamma^{\mu} \psi^{\Lambda}\right), \\
\mathcal{L}_{\mathrm{der}}^{N \Lambda}= & -\delta_{S}^{(N \Lambda)}\left(\partial_{\mu} \bar{\psi}^{N} \psi^{N}\right)\left(\partial^{\mu} \bar{\psi}^{\Lambda} \psi^{\Lambda}\right) \\
& -\delta_{V}^{(N \Lambda)}\left(\partial_{\mu} \bar{\psi}^{N} \gamma_{\nu} \psi^{N}\right)\left(\partial^{\mu} \bar{\psi}^{\Lambda} \gamma^{\nu} \psi^{\Lambda}\right), \\
\mathcal{L}_{\mathrm{ten}}^{N \Lambda}= & -\alpha_{T}^{(N \Lambda)}\left(\bar{\psi}^{\Lambda} \sigma^{\mu \nu} \psi^{\Lambda}\right)\left(\partial_{\mu} \bar{\psi}^{N} \gamma_{\nu} \psi^{N}\right) .
\end{aligned}
$$

We note that the vector-meson-like tensor coupling term, $\mathcal{L}_{\text {ten }}^{N \Lambda}$, is usually adopted to reproduce the smallness of spin-orbit splitting in $\Lambda$ single-particle spectra $[33,62-$ 67], although it can also be explained in terms of an almost complete cancelation between short-range scalar and vector contributions and longer range terms generated by two-pion exchange [37]. The Lagrangian contains sixteen coupling constants $\alpha_{S}, \alpha_{V}, \alpha_{T S}, \alpha_{T V}, \alpha_{S}^{(N \Lambda)}$, $\alpha_{V}^{(N \Lambda)}, \alpha_{T}^{(N \Lambda)}, \beta_{S}, \gamma_{S}, \gamma_{V}, \delta_{S}, \delta_{V}, \delta_{T S}, \delta_{T V}, \delta_{S}^{(N \Lambda)}$ and $\delta_{V}^{(N \Lambda)}$, which are usually optimized at the mean-field level to properties of several atomic nuclei and hypernuclei.

From the Lagrangian density (1), one can derive the corresponding energy $E_{\mathrm{RMF}}$ at the mean-field level with the no-sea approximation, which can be decomposed into two parts: the pure nuclear part and the $\Lambda$ hyperon part,

$$
E_{\mathrm{RMF}}=E_{\mathrm{RMF}}^{N}+E_{\mathrm{RMF}}^{\Lambda},
$$

with

$$
\begin{aligned}
& E_{\mathrm{RMF}}^{N}=T_{N}+\int d^{3} r\left[\varepsilon_{N N}(\mathbf{r})+\frac{1}{2} A_{0} e \rho_{V}^{(p)}\right], \\
& E_{\mathrm{RMF}}^{\Lambda}=T_{\Lambda}+\int d^{3} r \varepsilon_{N \Lambda}(\mathbf{r}) .
\end{aligned}
$$

The first term in these equations, $T_{B}=\operatorname{Tr}[(\boldsymbol{\alpha} \cdot \mathbf{p}+$ $\left.\left.\gamma^{0} m_{B}\right) \rho_{V}^{B}\right]$, is for the kinetic energy of nucleons $(B=N)$ or hyperon $(B=\Lambda)$, where $m_{B}$ is the corresponding mass. For the sake of simplicity, time-reversal invariance is usually imposed in the mean-field calculations for the $\Lambda$ hypernuclei, in which case, the $N N$ and $N \Lambda$ interaction terms are given by,

$$
\begin{aligned}
\varepsilon_{N N}= & \frac{1}{2} \sum_{m=S, V, T S, T V}\left[\alpha_{m}\left(\rho_{m}^{N}\right)^{2}+\delta_{m} \rho_{m} \Delta \rho_{m}^{N}\right] \\
& +\frac{1}{3} \beta_{S}\left(\rho_{S}^{N}\right)^{3}+\frac{1}{4} \gamma_{S}\left(\rho_{S}^{N}\right)^{4}+\frac{1}{4} \gamma_{V}\left(\rho_{V}^{N}\right)^{4} \\
\varepsilon_{N \Lambda}= & \sum_{m=S, V}\left[\alpha_{m}^{(N \Lambda)} \rho_{m}^{N} \rho_{m}^{\Lambda}+\delta_{m}^{(N \Lambda)} \rho_{m}^{N} \Delta \rho_{m}^{\Lambda}\right] \\
& +\alpha_{T}^{(N \Lambda)} \rho_{V}^{N} \rho_{T}^{\Lambda},
\end{aligned}
$$

respectively. In these equations, the densities $\rho_{m}^{B}$ and the tensor density $\rho_{T}^{\Lambda}$ are defined as

$$
\rho_{m}^{B}=\sum_{k} v_{k}^{2} \bar{\psi}_{k}^{B} \Gamma_{m} \psi_{k}^{B}, \quad \rho_{T}^{\Lambda}=\nabla \cdot\left(\bar{\psi}^{\Lambda} i \boldsymbol{\alpha} \psi^{\Lambda}\right),
$$

where the vertex $\Gamma_{m}$ is $1, \gamma^{0}, \tau_{3}$, and $\gamma^{0} \tau_{3}$, with the index $m$ running over $S, V, T S$ and $T V$, which represents respectively the isoscalar-scalar, isoscalar-vector, isovectorscalar and isovector-vector types of coupling characterized by their transformation properties in isospin-Lorentz spaces. $v_{k}^{2}$ is the occupation probability of the $k$-th singleparticle energy level of neutrons or protons to be determined by the BCS method. The $\boldsymbol{\alpha}$ and $\gamma^{\mu}$ are the $4 \times 4$ Dirac matrices.

Minimization of the RMF energy (4) with respect to the single-particle wave function for nucleons or hyperon leads to the Dirac equation, which reads,

$$
\left[\boldsymbol{\alpha} \cdot \mathbf{p}+V_{0}^{N}+\gamma^{0}\left(m_{N}+S^{N}\right)\right] \psi_{k}^{N}(\boldsymbol{r})=\epsilon_{k}^{N} \psi_{k}^{N}(\boldsymbol{r}),
$$

for nucleons, with the scalar field $S^{N}(\boldsymbol{r})=\Sigma_{S}(\boldsymbol{r})+$ $\tau_{3} \Sigma_{T S}(\boldsymbol{r})$ and vector field $V_{0}^{N}(\boldsymbol{r})=\Sigma_{V}(\boldsymbol{r})+\tau_{3} \Sigma_{T V}(\boldsymbol{r})$ defined as

$$
\begin{array}{rlr}
\Sigma_{S}= & \alpha_{S} \rho_{S}^{N}+\beta_{S}\left(\rho_{S}^{N}\right)^{2}+\gamma_{S}\left(\rho_{S}^{N}\right)^{3}+\delta_{S} \Delta \rho_{S}^{N} & (9 \mathrm{a}) \\
& +\alpha_{S}^{(N \Lambda)} \rho_{S}^{\Lambda}+\delta_{S}^{(N \Lambda)} \Delta \rho_{S}^{\Lambda}, & (9 \mathrm{~b}) \\
\Sigma_{T S}= & \delta_{T S} \Delta \rho_{T S}^{N}+\alpha_{T S} \rho_{T S}^{N}, \\
\Sigma_{V}= & \alpha_{V} \rho_{V}^{N}+\gamma_{V}\left(\rho_{V}^{N}\right)^{3}+\delta_{V} \Delta \rho_{V}^{N}+e A_{0} \frac{1-\tau_{3}}{2}(9 \mathrm{~d}) \\
& +\alpha_{V}^{(N \Lambda)} \rho_{V}^{\Lambda}+\delta_{V}^{(N \Lambda)} \Delta \rho_{V}^{\Lambda}+\alpha_{T}^{(N \Lambda)} \rho_{T}^{\Lambda}, \\
\Sigma_{T V}= & \alpha_{T V} \rho_{T V}^{N}+\delta_{T V} \Delta \rho_{T V}^{N} .
\end{array}
$$

On the other hand, the Dirac equation for the $\Lambda$ hyperon inside the hypernucleus reads

$$
\left[\boldsymbol{\alpha} \cdot \mathbf{p}+V_{0}^{\Lambda}+\gamma^{0}\left(S^{\Lambda}+m_{\Lambda}\right)\right] \psi_{k}^{\Lambda}(\boldsymbol{r})=\epsilon_{k}^{\Lambda} \psi_{k}^{\Lambda}(\boldsymbol{r})
$$

with the vector field $V_{0}^{\Lambda}=U_{V}+U_{T}$, and

$$
\begin{aligned}
U_{V} & =\delta_{V}^{(N \Lambda)} \Delta \rho_{V}^{N}+\alpha_{V}^{(N \Lambda)} \rho_{V}^{N}, \\
U_{T} & =-i \alpha_{T}^{(N \Lambda)} \beta \boldsymbol{\alpha} \cdot \nabla \rho_{V}^{N}, \\
S^{\Lambda} & =\delta_{S}^{(N \Lambda)} \Delta \rho_{S}^{N}+\alpha_{S}^{(N \Lambda)} \rho_{S}^{N} .
\end{aligned}
$$

These two Dirac equations, Eqs. (8) and (10), are solved by expanding the Dirac spinors $\psi_{k}^{B}$ for nucleons and hyperon on the basis of a $3 \mathrm{DHO}$ with the oscillator length parameter chosen as $b_{x}=b_{y}=b_{z}=\sqrt{\hbar / m \omega_{0}}$, where the oscillator frequency is determined by $\hbar \omega_{0}=$ $41 A^{-1 / 3}(\mathrm{MeV})$. In addition, to reduce the computational task, it is assumed that the total densities are symmetric under reflections with respect to the three planes $x y$, $x z$ and $y z$. The Coulomb field $A_{0}$ is obtained through a direct integration of the Poisson equation. To obtain the total energies and the mean-field wave functions for triaxially deformed hypernuclei and the corresponding core nucleus as a function of deformation parameters $(\beta, \gamma)$, a 
quadratic constraint calculation on the mass quadrupole moments is carried out by minimizing the following energy with respect to single-particle wave function,

$$
E^{\prime}=E_{\mathrm{RMF}}+\sum_{\mu=0,2} C_{2 \mu}\left(\left\langle\hat{Q}_{2 \mu}\right\rangle-q_{2 \mu}\right)^{2}
$$

where $C_{2 \mu}$ is a stiffness parameter and $\left\langle\hat{Q}_{2 \mu}\right\rangle$ denotes the expectation value of the mass quadrupole moment operator,

$$
\hat{Q}_{20}=\sqrt{\frac{5}{16 \pi}}\left(2 z^{2}-x^{2}-y^{2}\right), \quad \hat{Q}_{22}=\sqrt{\frac{15}{32 \pi}}\left(x^{2}-y^{2}\right) .
$$

In Eq. (12), $q_{2 \mu}$ are the quadrupole moment of meanfield state to be obtained. The deformation parameters $(\beta, \gamma)$ of mean-field state are related to the expectation values of the mass quadrupole moment operator by $\beta=$ $\frac{4 \pi}{3 A R^{2}} \sqrt{\left\langle Q_{20}\right\rangle^{2}+2\left\langle Q_{22}\right\rangle^{2}}$ and $\gamma=\tan ^{-1}\left(\sqrt{2} \frac{Q_{22}}{Q_{20}}\right)$, respectively, with $R=1.2 A^{1 / 3}$ (fm). We note that the deformation parameters $(\beta, \gamma)$ are calculated with the nuclear density $\rho^{N}(\boldsymbol{r})$ for the core nuclei (cn) and with the total density $\rho^{N}(\boldsymbol{r})+\rho^{\Lambda}(\boldsymbol{r})$ for the hypernuclei.

The center-of-mass correction energy $E_{\mathrm{cm}}$ is calculated by taking the expectation value of the kinetic energy for the center-of-mass motion with respect to the mean-field wave function. For a single- $\Lambda$ hypernucleus, it is given by

$$
E_{\mathrm{cm}}=\frac{\left\langle\mathbf{P}_{N}^{2}+\mathbf{P}_{\Lambda}^{2}\right\rangle}{2\left(A m_{N}+m_{\Lambda}\right)}
$$

where $\mathbf{P}_{B}$ is the total momentum of the baryons $(B=$ $N, \Lambda)$ in hypernucleus with $A$ nucleons and one $\Lambda$ hyperon.

Following Refs. [46, 60], the pairing correlation among nucleons is taken into account with the BCS method using a density-independent zero-range pairing force supplemented with a smooth cutoff [68]. The resultant pairing energy $E_{\text {pair }}$ is added to the total energy, which is finally given by

$$
E_{\mathrm{tot}}\left({ }_{\Lambda}^{A+1} Z\right)=E_{\mathrm{RMF}}-A m_{N} c^{2}-m_{\Lambda} c^{2}-E_{\mathrm{cm}}+E_{\mathrm{pair}} .
$$

The total binding energy $B\left({ }_{\Lambda}^{A+1} Z\right)$ of a single- $\Lambda$ hypernucleus is given by $B\left({ }_{\Lambda}^{A+1} Z\right)=-E_{\text {tot }}\left({ }_{\Lambda}^{A+1} Z\right)$. To study the change of energy surface of nuclear core by the $\Lambda$ hyperon, we also introduce the energy $E_{\text {tot }}^{\mathrm{cn}}\left({ }_{\Lambda}^{A+1} Z\right)$ for the core nucleus inside a hypernucleus as

$$
E_{\mathrm{tot}}^{\mathrm{cn}}\left({ }_{\Lambda}^{A+1} Z\right) \equiv E_{\mathrm{tot}}\left({ }_{\Lambda}^{A+1} Z\right)-E_{\mathrm{RMF}}^{\Lambda}+E_{\mathrm{cm}}^{\Lambda},
$$

where the last term, $E_{\mathrm{cm}}^{\Lambda}=\frac{\left\langle\mathbf{P}_{\Lambda}^{2}\right\rangle}{2\left(A m_{N}+m_{\Lambda}\right)}$, is the contribution of $\Lambda$ particle to the center-of-mass correction energy.

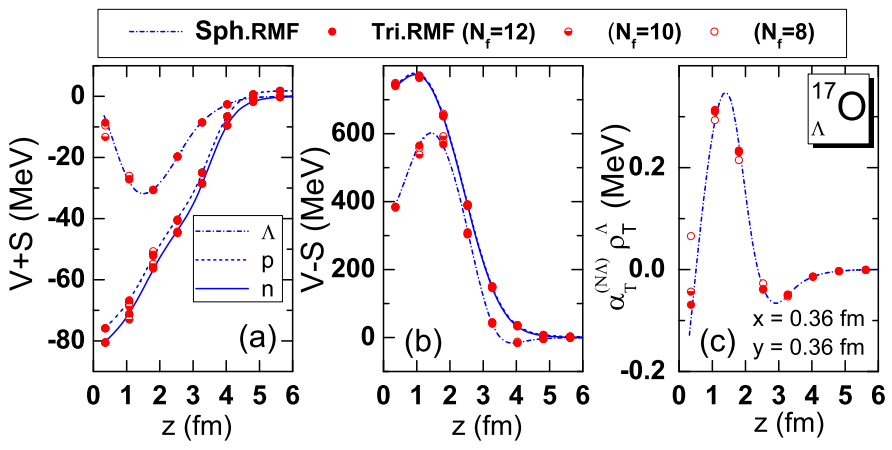

FIG. 1: (Color online) The convergence feature of potentials $V_{0}^{B}(\boldsymbol{r})+S^{B}(\boldsymbol{r})(\mathrm{a}), V_{0}^{B}(\boldsymbol{r})-S^{B}(\boldsymbol{r})$ (b) (with $B=n, p, \Lambda$ ) and the tensor density $\alpha_{T}^{(N \Lambda)} \rho_{T}^{\Lambda}(\boldsymbol{r})$ (c) with respect to the maximum number of major shell $\left(N_{f}\right)$ in the $3 \mathrm{DHO}$ basis for the triaxial RMF calculation for ${ }_{\Lambda}^{17} \mathrm{O}$. These quantities are evaluated at $x=y=0.36 \mathrm{fm}$ and plotted as a function of $z$. For a comparison, the results of spherical RMF calculations are also shown by the lines.

\section{RESULTS OF MEAN-FIELD STUDIES}

\section{A. Illustrative calculations}

We first carry out an illustrative calculation to test our triaxial RMF code for some spherical hypernuclei, comparing with the results by the spherical RMF code in coordinate space with the box size of $R=15 \mathrm{fm}$ [62]. If not particularly indicated, the same parameters are used in both calculations, including the masses of nucleons and $\Lambda$ which are taken as $m_{N}=939 \mathrm{MeV} / c^{2}$ and $m_{\Lambda}=1115.6 \mathrm{MeV} / c^{2}$, respectively and the PC-F1 force [60] for the $N N$ interaction and the PCY-S1 [62] for the $N \Lambda$ interaction. Taking ${ }_{\Lambda s}^{17} \mathrm{O}$ as an example, where the $\Lambda$ particle is put in the $1 s_{1 / 2}$ orbital, we plot in Fig. 1 the mean-field potentials $V_{0}^{B}+S^{B}$ and $V_{0}^{B}-S^{B}$ for neutron, proton and $\Lambda$, together with the tensor density $\alpha_{T}^{(N \Lambda)} \rho_{T}^{\Lambda}$ from the triaxial RMF calculation. We compare the results with three different maximum major-shell numbers in the $3 \mathrm{DHO}$ basis, $N_{f}=8,10$, and 12 . The results are compared also with those by the spherical RMF calculations. It is shown that the mean-field potentials are well converged at $N_{f}=10$. In contrast, the tensor density (7) originated from the tensor coupling term $\mathcal{L}_{\text {ten }}^{N \Lambda}$ has a slightly slower convergence behavior, especially at small values of $z$. It is shown that the tensor density obtained with the maximum shell number $N_{f}=12$ gives a good agreement with the spherical RMF result.

Table I shows the detailed structural properties of hypernuclei, ${ }_{\Lambda s}^{17} \mathrm{O},{ }_{\Lambda s}^{31} \mathrm{Si},{ }_{\Lambda s}^{33} \mathrm{~S}$, and ${ }_{\Lambda s}^{41} \mathrm{Ca}$ from both the spherical and triaxial RMF calculations. In the triaxial RMF calculation, fourteen major $\mathrm{HO}$ shells $\left(N_{f}=14\right)$ are adopted to expand the Dirac spinors, with which the quadrupole deformation $\beta$ of hypernuclei is convergent to zero. It is shown that both approaches give the results very close to each other. The remaining small differ- 
TABLE I: The total energy $E_{\text {tot }}$, the kinetic energy $E_{\text {kin }}\left(=T_{N}+T_{\Lambda}\right)$, the root-mean-square (rms) radii of neutrons $r_{n}$, protons $r_{p}$ and hyperon $r_{\Lambda}$, and the energy of the lowest three single-particle states for neutron, proton and hyperon obtained with the triaxial RMF (Tri.RMF) calculation for ${ }_{\Lambda s}^{17} \mathrm{O},{ }_{\Lambda s}^{31} \mathrm{Si},{ }_{\Lambda s}^{33} \mathrm{~S}$, and ${ }_{\Lambda s}^{41} \mathrm{Ca}$, in comparison with those with the spherical RMF (Sph.RMF) calculations. All the energies are in $\mathrm{MeV}$ and the radii are in fm.

\begin{tabular}{|c|c|c|c|c|c|c|c|c|c|}
\hline & & \multicolumn{2}{|c|}{${ }_{\Lambda s}^{17} \mathrm{O}$} & \multicolumn{2}{|c|}{$\begin{array}{l}{ }_{\Lambda s}^{31} \mathrm{Si} \\
\end{array}$} & \multicolumn{2}{|c|}{$\begin{array}{c}33 \\
\Lambda_{s} \mathrm{~S} \\
\end{array}$} & \multicolumn{2}{|c|}{$\begin{array}{c}{ }_{\Lambda s} \mathrm{Ca} \\
\end{array}$} \\
\hline & & Sph.RMF & Tri.RMF & Sph.RMF & Tri.RMF & Sph.RMF & Tri.RMF & Sph.RMF & Tri.RMF \\
\hline & $E_{\text {tot }}$ & -140.317 & -140.309 & -269.491 & -269.476 & -285.434 & -285.320 & -363.459 & -363.174 \\
\hline & $E_{\text {kin }}$ & 210.036 & 210.031 & 435.385 & 435.436 & 439.216 & 439.164 & 518.686 & 518.239 \\
\hline & $E_{\mathrm{cm}}$ & 9.752 & 9.750 & 9.282 & 9.281 & 8.915 & 8.902 & 8.167 & 8.140 \\
\hline & $r_{n}$ & 2.613 & 2.613 & 3.084 & 3.083 & 3.088 & 3.088 & 3.340 & 3.341 \\
\hline & $r_{p}$ & 2.638 & 2.638 & 2.984 & 2.984 & 3.129 & 3.129 & 3.385 & 3.386 \\
\hline & $r_{\Lambda}$ & 2.458 & 2.458 & 2.516 & 2.515 & 2.571 & 2.570 & 2.820 & 2.823 \\
\hline & $1 \mathrm{~s}_{1 / 2}$ & -41.629 & -41.628 & -54.234 & -54.260 & -57.544 & -57.528 & -53.827 & -53.817 \\
\hline neutron & $1 \mathrm{p}_{3 / 2}$ & -21.937 & -21.937 & -34.531 & -34.551 & -36.477 & -36.480 & -37.859 & -37.854 \\
\hline & $1 \mathrm{p}_{1 / 2}$ & -15.285 & -15.288 & -27.694 & -27.728 & -28.480 & -28.496 & -33.354 & -33.362 \\
\hline & $1 \mathrm{~s}_{1 / 2}$ & -37.517 & -37.514 & -51.510 & -51.521 & -50.462 & -50.445 & -45.721 & -45.712 \\
\hline proton & $1 \mathrm{p}_{3 / 2}$ & -18.107 & -18.105 & -30.474 & -30.490 & -29.744 & -29.746 & -30.102 & -30.096 \\
\hline & $1 \mathrm{p}_{1 / 2}$ & -11.531 & -11.533 & -23.848 & -23.877 & -21.827 & -21.841 & -25.601 & -25.608 \\
\hline & $1 \mathrm{~s}_{1 / 2}$ & -12.569 & -12.570 & -18.908 & -18.946 & -18.458 & -18.483 & -18.305 & -18.278 \\
\hline hyperon & $1 p_{3 / 2}$ & -2.336 & -2.297 & -8.485 & -8.510 & -8.405 & -8.416 & -10.112 & -10.108 \\
\hline & $1 \mathrm{p}_{1 / 2}$ & -1.995 & -1.947 & -8.391 & -8.406 & -8.189 & -8.207 & -10.255 & -10.263 \\
\hline
\end{tabular}

ences in the binding energies can be reduced further by increasing the maximum major-shell number $N_{f}$ of the $\mathrm{HO}$ basis and constraining the high-order hexadecapole deformation to be zero in the triaxial RMF calculation.

We note that the results presented in Tab. I are obtained without breaking the symmetry of time-reversal invariance in the mean-field calculations for the $\Lambda$ hypernuclei. It has been found in recent studies $[69,70]$ that the effect of time-odd fields from the breaking of time-reversal invariance by one unpaired nucleon on the binding energies is in between 0.1 and $0.2 \mathrm{MeV}$ for the ground state of the sd-shell odd-mass nuclei. For the single- $\Lambda$ hypernuclei with an even-even nuclear core and the $\Lambda$ in a low orbital-angular-momentum state, the effect caused by the unpaired $\Lambda$ hyperon is even smaller and it leads to an energy splitting of $0.1 \mathrm{MeV}$ for the time-reversal partner states of $s_{1 / 2}$ orbital in ${ }_{\Lambda}^{17} \mathrm{O}$ [56]. Since the time-reversal invariance is imposed in both the spherical and triaxial deformed RMF calculations, this effect will not contribute to the difference in the two results.

\section{B. Hyperon binding energy and deformation effect}

We first discuss the effect of deformation on the binding energy of hypernuclei. Figure 2(a) shows the $\Lambda$ binding energies $B_{\Lambda}$ in single- $\Lambda$ hypernuclei obtained with the spherical and triaxial RMF calculations, in comparison with the experimental data. Here, $B_{\Lambda}$ is defined as the energy difference between the ground state (g.s.) of hypernucleus and that of the core nucleus, that is, $B_{\Lambda} \equiv E_{\text {tot }}\left({ }^{A} Z, g . s.\right)-E_{\text {tot }}\left({ }_{\Lambda}^{A+1} Z, g . s.\right)$. In the triaxial

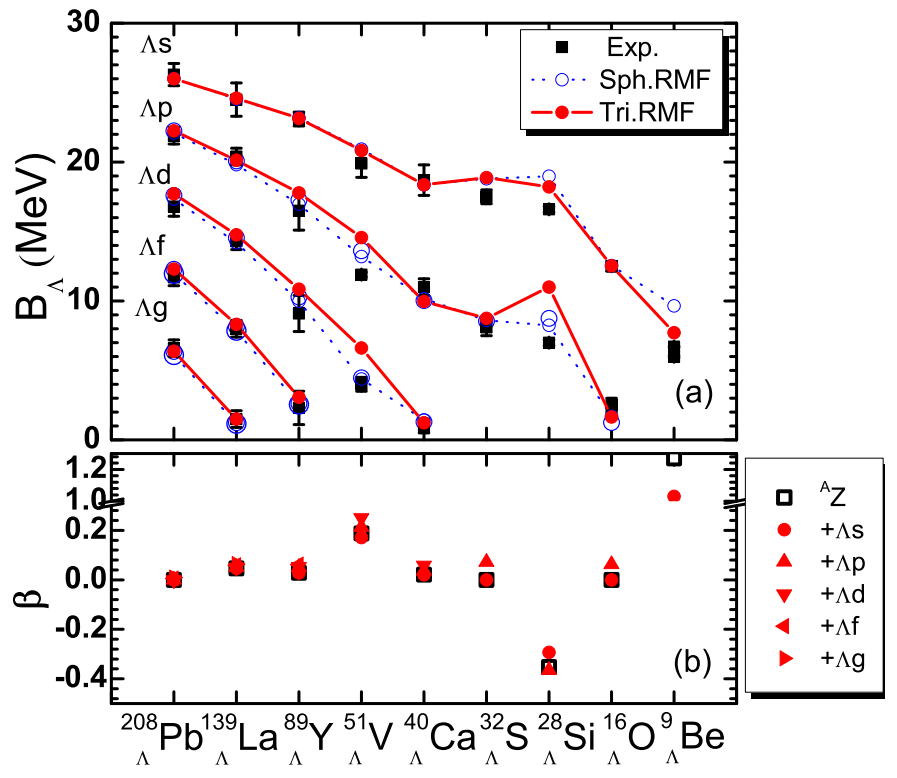

FIG. 2: (Color online) (a) The $\Lambda$ binding energies in single- $\Lambda$ hypernuclei with $\Lambda$ in different single-particle states obtained with the spherical and the triaxial RMF calculations, and their comparison with the available data from Refs. [20, 7173]. The parameter set PC-F1 and PCY-S1 are adopted for the $N N$ and the $N \Lambda$ interactions, respectively. (b) The quadrupole deformation $\beta$ for each $\Lambda$ hypernuclei obtained with the triaxial RMF calculation. See text for more details.

RMF calculation for the $\Lambda$ hypernuclei, the $\Lambda$ hyperon is always put in the lowest state among those which are connected to the $s, p, \ldots, g$ state in the spherical limit. The corresponding $\Lambda$ is therefore denoted as $\Lambda_{s}, \Lambda_{p}, \ldots, \Lambda_{g}$ 


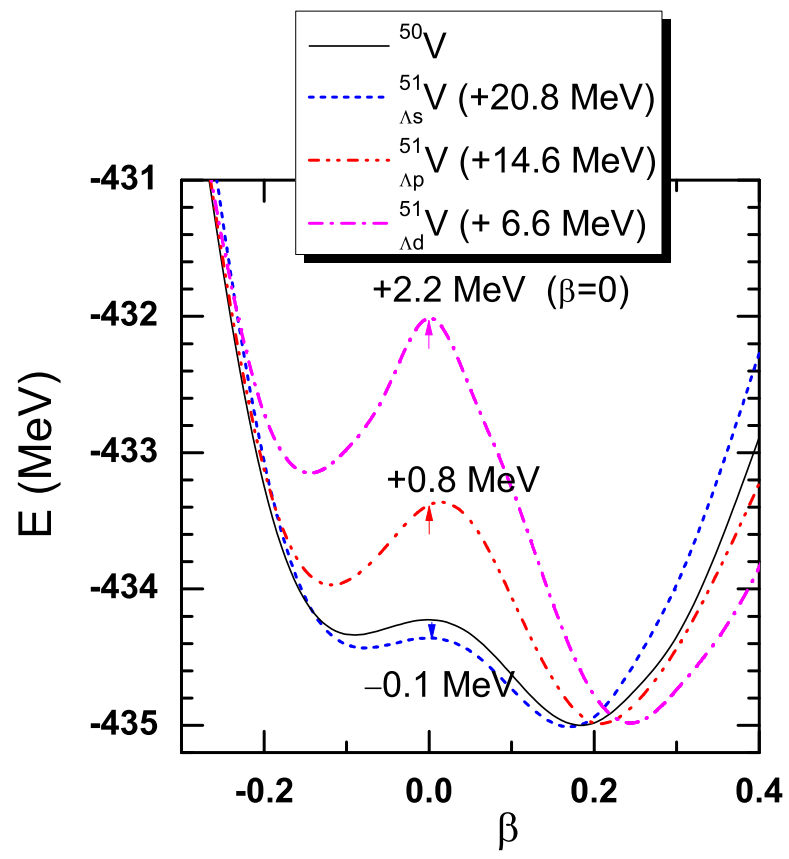

FIG. 3: (Color online) The total energy of ${ }^{50} \mathrm{~V},{ }_{\Lambda s}^{51} \mathrm{~V},{ }_{\Lambda p}^{51} \mathrm{~V}$ and ${ }_{\Lambda d}^{51} \mathrm{~V}$ as a function of deformation parameter $\beta$ with the triaxially deformed RMF calculations. The energy of hypernuclei is shifted by normalizing the minimum energy to that of ${ }^{50} \mathrm{~V}$. The energy difference between the hypernuclei and ${ }^{50} \mathrm{~V}$ at $\beta=0$ is indicated with the numbers.

for convenience. In the spherical RMF calculations, two values of $B_{\Lambda}$ for the $\ell_{\Lambda} \neq 0$ cases are plotted, corresponding to the $\Lambda$ hyperon in the spin-orbit partner states. Due to the introduction of a strong $\Lambda$ tensor coupling, the energy splitting of the spin-orbit partner states by the spherical RMF calculation is less than $0.5 \mathrm{MeV}$ and mostly with an opposite sign to the ordinary nuclei [62]. The triaxial RMF approach yields similar results as the spherical RMF approach for the $\Lambda$ binding energies of most hypernuclei, except for ${ }_{\Lambda}^{9} \mathrm{Be},{ }_{\Lambda}^{28} \mathrm{Si}$ and ${ }_{\Lambda}^{51} \mathrm{~V}$ which are deformed in their ground states (See Fig. 2(b) for the value of deformation parameter). For these nuclei, a nonzero deformation $\beta$ decreases the binding energy of $\Lambda_{s}$ and improves the agreement with the data, while it signinificantly overestimates the binding energy of $\Lambda_{p}$ and $\Lambda_{d}$.

We take hypernucleus ${ }_{\Lambda}^{51} \mathrm{~V}$ as an example to illustrate the deformation effect on $B_{\Lambda}$. Figure 3 shows that the energy minimum of hypernucleus ${ }_{\Lambda s}^{51} \mathrm{~V}$ is shifted slightly towards spherical shape, while that of ${ }_{\Lambda p}^{51} \mathrm{~V}$ and ${ }_{\Lambda d}^{51} \mathrm{~V}$ is pushed to a larger deformed shape. Moreover, it is shown that the deformation of hypernuclei increases from ${ }_{\Lambda s}^{51} \mathrm{~V}$ to ${ }_{\Lambda p}^{51} \mathrm{~V}$, and then to ${ }_{\Lambda d}^{51} \mathrm{~V}$. The difference in the $B_{\Lambda}$ values of ${ }_{\Lambda}^{51} \mathrm{~V}$ by the spherical and triaxial RMF calculations is also shown clearly in Fig. 3, where the energy of hypernucleus ${ }_{\Lambda}^{51} \mathrm{~V}$ decreases or increases by $0.1 \mathrm{MeV}, 0.8 \mathrm{MeV}$ and $2.2 \mathrm{MeV}$ for the $\Lambda_{s}, \Lambda_{p}$ and $\Lambda_{d}$, respectively. The microscopic mechanism responsible for these phenomena

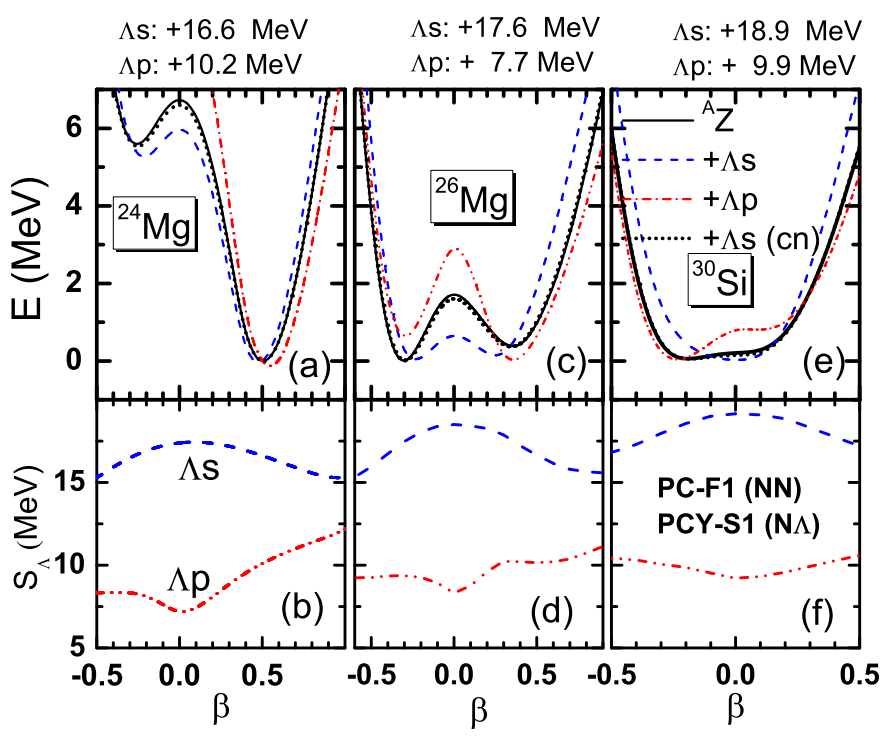

FIG. 4: (Color online) The potential energy surfaces obtained with the triaxially deformed RMF calculations for (a) ${ }^{24} \mathrm{Mg}$, ${ }_{\Lambda s}^{25} \mathrm{Mg},{ }_{\Lambda p}^{25} \mathrm{Mg}$ and the core nucleus inside ${ }_{\Lambda s}^{25} \mathrm{Mg}$; (c) ${ }^{26} \mathrm{Mg}$, ${ }_{\Lambda s}^{27} \mathrm{Mg},{ }_{\Lambda p}^{27} \mathrm{Mg}$ and the core nucleus inside ${ }_{\Lambda s}^{27} \mathrm{Mg}$; (e) ${ }^{30} \mathrm{Si},{ }_{\Lambda s}^{31} \mathrm{Si}$, ${ }_{\Lambda p}^{31} \mathrm{Si}$ and the core nucleus inside ${ }_{\Lambda s}^{30} \mathrm{Si}$ as a function of deformation parameter $\beta$. All the energies are normalized to the global minimum. The deformation-dependent $\Lambda$ separation energy defined by Eq. (17) as a function of deformation parameter $\beta$ for (b) ${ }_{\Lambda s}^{25} \mathrm{Mg}$ and ${ }_{\Lambda p}^{25} \mathrm{Mg}$; (d) ${ }_{\Lambda s}^{27} \mathrm{Mg}$ and ${ }_{\Lambda p}^{27} \mathrm{Mg}$; and (f) ${ }_{\Lambda s}^{31} \mathrm{Si}$ and ${ }_{\Lambda p}^{31} \mathrm{Si}$. The binding energies of $\Lambda_{s}$ and $\Lambda_{p}$ for each case are given on the top of the figures.

can be traced to the Nilsson diagram of hyperon singleparticle energies, which will be discussed in details in the next subsection.

\section{Shape polarization effect of $\Lambda$ hyperon in $s d$-shell nuclei in $(\beta, \gamma)$ plane}

In order to discuss more in details the shape polarization effect of $\Lambda$ hyperon, we take as examples three $s d$-shell hypernuclei, the prolate deformed ${ }^{24} \mathrm{Mg}$ and the oblate deformed ${ }^{26} \mathrm{Mg}$ and ${ }^{30} \mathrm{Si}$, the latter two having transitional characters. Figure 4 (a) shows the PESs for the ${ }_{\Lambda s}^{25} \mathrm{Mg},{ }_{\Lambda p}^{25} \mathrm{Mg},{ }_{\Lambda s}^{27} \mathrm{Mg},{ }_{\Lambda p}^{27} \mathrm{Mg},{ }_{\Lambda s}^{31} \mathrm{Si}$, and ${ }_{\Lambda p}^{31} \mathrm{Si}$ obtained with the triaxial RMF method as a function of deformation parameter $\beta$. The energy surfaces for the corresponding core nuclei are also shown. As in the case of ${ }_{\Lambda}^{51} \mathrm{~V}$ shown in Fig. 3, the energy minimum of ${ }_{\Lambda s}^{25} \mathrm{Mg}$ and ${ }_{\Lambda p}^{25} \mathrm{Mg}$ is shifted to a slightly smaller and larger deformed region, respectively, compared with that of the core nucleus. For ${ }^{26} \mathrm{Mg}$, the $\Lambda_{s}$ significantly lowers down the barrier between the oblate and prolate minima. Of particular interest is that the $\Lambda_{p}$ inverts the energy order of the oblate and prolate minima in ${ }^{26} \mathrm{Mg}$. A significant change of the deformation parameter of the mean-field ground state by $\Lambda$ hyperon is shown in ${ }^{30} \mathrm{Si}$. That is, the $\Lambda_{s}$ brings the oblate deformed ${ }^{30} \mathrm{Si}$ to spherical ${ }_{\Lambda s}^{31} \mathrm{Si}$. A sim- 
ilar conclusion has been obtained also in Refs. $[9,10]$. In contrast, the $\Lambda_{p}$ drives ${ }^{30} \mathrm{Si}$ to be more oblate deformed. It is worthwhile to mention that although the PESs of hypernuclei could be significantly different from those of core nuclei, the differences in the PESs for the core nuclei inside the hypernuclei (the dotted lines) and for the core nuclei without the hyperon impurity (the solid lines) are negligibly small.

To illustrate the shape-driving effect of $\Lambda$ hyperon in different orbitals, we introduce a deformation-dependent $\Lambda$ separation energy $S_{\Lambda}(\beta)$ as

$$
S_{\Lambda}(\beta) \equiv E_{\mathrm{tot}}\left({ }^{A} Z, \beta\right)-E_{\mathrm{tot}}\left({ }_{\Lambda}^{A+1} Z, \beta\right),
$$

where $E_{\text {tot }}\left({ }_{\Lambda}^{A+1} Z, \beta\right)$ and $E_{\text {tot }}\left({ }^{A} Z, \beta\right)$ are the total energies of the hypernucleus and the core nucleus at deformation $\beta$, respectively. Here, we take the same deformation value $\beta$ for the hypernucleus and the core nucleus to define the quantity $S_{\Lambda}(\beta)$. Notice that even though $S_{\Lambda}(\beta)$ is different from the standard definition of hyperon separation energy, it provides a convenient way to understand the shape polarization effect of $\Lambda$. The $S_{\Lambda}(\beta)$ in ${ }_{\Lambda s}^{25} \mathrm{Mg}$, ${ }_{\Lambda p}^{25} \mathrm{Mg},{ }_{\Lambda s}^{27} \mathrm{Mg},{ }_{\Lambda p}^{27} \mathrm{Mg},{ }_{\Lambda s}^{31} \mathrm{Si}$, and ${ }_{\Lambda p}^{31} \mathrm{Si}$ is shown in Figs. 4 (b), (d) and (f) as a function of deformation $\beta$. It is seen clearly that the $S_{\Lambda}$ decreases (or increases) with $\beta$ for $\Lambda_{s}$ (or $\Lambda_{p}$ ), which provides a mechanism to change the structure of the PESs in such a way that the nuclear shape becomes less or more deformed.

Figure 5(a) shows a comparison of the potential energy surfaces for ${ }^{30} \mathrm{Si},{ }_{\Lambda s}^{31} \mathrm{Si}$, and ${ }_{\Lambda p}^{31} \mathrm{Si}$ from the deformed RMF calculation with different $N \Lambda$ interactions, namely the PCY-S1 and PCY-S4 [62]. It is seen that the potential energy surface of ${ }_{\Lambda s}^{31} \mathrm{Si}$ is similar to each other for both of the two $N \Lambda$ interactions. However, that of ${ }_{\Lambda p}^{31} \mathrm{Si}$ are evidently different in some deformation region. This difference is shown more clearly in the comparison of $\Lambda$ separation energy $S_{\Lambda}(\beta)$ in Fig. $5(\mathrm{~b})$. For $\Lambda_{s}$, the two $N \Lambda$ interactions give similar slops of $S_{\Lambda}(\beta)$ as a function of deformation $\beta$. On the other hand, for $\Lambda_{p}$, the slop is apparently different on the prolate side, resulting in the different behavior of the potential energy surface of ${ }_{\Lambda p}^{31} \mathrm{Si}$. It implies that the impurity effect of $\Lambda_{p}$ is somewhat sensitive to the $N \Lambda$ interaction, in contrast to that of $\Lambda_{s}$.

The influence of $\Lambda$ hyperon on nuclear triaxiality is illustrated in Fig. 6, which shows the PESs for the ${ }_{\Lambda s}^{25} \mathrm{Mg}$, ${ }_{\Lambda p}^{25} \mathrm{Mg},{ }_{\Lambda s}^{27} \mathrm{Mg}$, and ${ }_{\Lambda p}^{27} \mathrm{Mg}$, and their core nuclei as a function of $\gamma$ deformation. For ${ }^{24} \mathrm{Mg}$ with a pronounced prolate energy minimum, the stiffness of the PES along $\gamma$ deformation increases by adding $\Lambda_{s}$ or $\Lambda_{p}$. This is an opposite tendency from that predicted by the SkyrmeHartree-Fock (SHF) calculation [40]. For ${ }^{26} \mathrm{Mg}$, with a shallow oblate energy minimum, the inclusion of $\Lambda_{s}$ softens the PES along $\gamma$ deformation, in agreement with the previous SHF calculation. However, at the same time, the energy minimum is shifted to a prolate-like shape with $\gamma=12^{\circ}$ by adding $\Lambda_{p}$, which is a consequence of competition between the $\gamma$ deformation effect and the $\Lambda_{p}$ impurity effect.

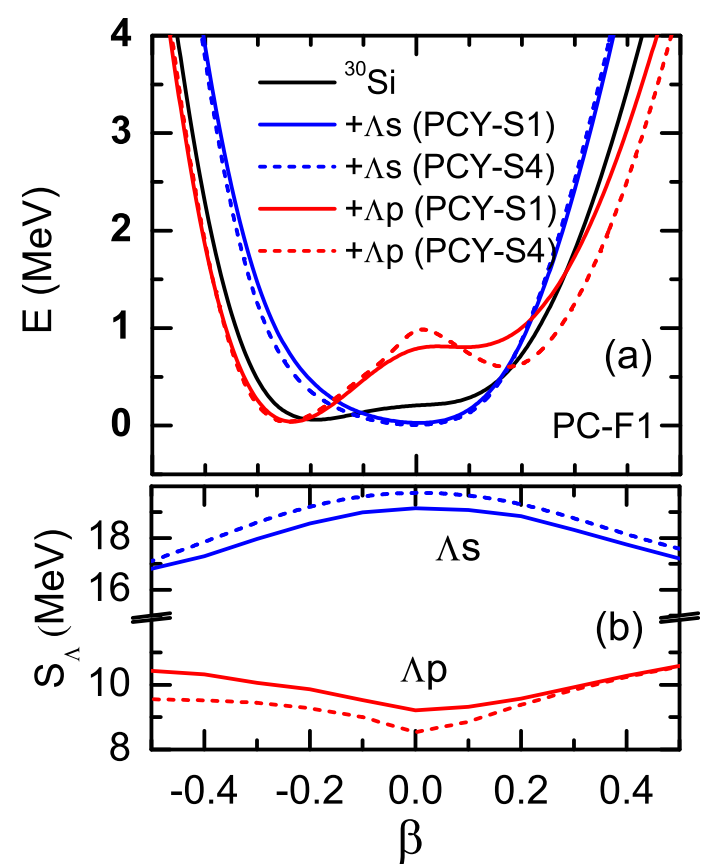

FIG. 5: (Color online) (a) The potential energy surfaces for ${ }^{30} \mathrm{Si},{ }_{\Lambda s}^{31} \mathrm{Si},{ }_{\Lambda p}^{31} \mathrm{Si}$ as a function of deformation parameter $\beta$ obtained with the deformed RMF calculations using the same PC-F1 force for the $N N$ interaction but different $N \Lambda$ interaction (PCY-S1 and PCY-S4 [62], respectively). The energies are normalized to the global minima. (b) The $\Lambda$ separation energy $S_{\Lambda}(\beta)$ as a function of deformation parameter $\beta$.

The impurity effects of $\Lambda_{s}$ and $\Lambda_{p}$ on nuclear quadrupole deformation $\beta$ and $\gamma$ discussed in Figs. 4 and 6 can be also studied in the PES in the whole $(\beta, \gamma)$ plane. Figs. 7, 8 and 9 show the PESs for ${ }^{24,26} \mathrm{Mg}$ and ${ }^{30} \mathrm{Si}$, together with the hypernuclei ${ }^{25,27} \mathrm{Mg}$ and ${ }_{\Lambda}^{31} \mathrm{Si}$ with a $\Lambda_{s}$ or $\Lambda_{p}$. The contribution of $\Lambda_{s}$ and $\Lambda_{p}$ to the total energy of hypernuclei in $(\beta, \gamma)$ plane is also plotted. One can again see that the $\Lambda_{s}$ stabilizes the spherical shape, while the $\Lambda_{p}$ stabilizes deformed shape.

To understand the impurity effects of $\Lambda_{s}$ and $\Lambda_{p}$ in a qualitative way, we plot in Fig. 10 the Nilsson diagram of the single-particle energy for $\Lambda$ hyperon in ${ }_{\Lambda s}^{25} \mathrm{Mg}$ as a function of deformation parameters $\beta$ and $\gamma$. The results of the calculation without the tensor potential $U_{T}$ in the Dirac equation for $\Lambda$ hyperon are also plotted for comparison. It is shown that the tensor potential $U_{T}$ pushes up the energy of $1 s_{1 / 2}$ and $1 p_{3 / 2}$ orbitals and reduces significantly the spin-orbit splitting between the partner states $\Lambda p_{3 / 2}$ and $\Lambda p_{1 / 2}$ at the spherical shape. The resultant splitting energy is $\Delta E_{\mathrm{so}}=\epsilon_{j=l-1 / 2}-\epsilon_{j=l+1 / 2}=-0.16$ $\mathrm{MeV}$. The inversion of the energy order of the spin-orbit partner states for $\Lambda$ hyperon is a particular character of the parameter set PCY-S1 for $N \Lambda$ interaction with a very strong tensor coupling [62].

With the increase of deformation $\beta$, the $\Lambda_{s}$ becomes slightly less bound, while the $\Lambda_{p}$ becomes deeper bound. It explains both the behaviors of $\Lambda$ separation energy as 


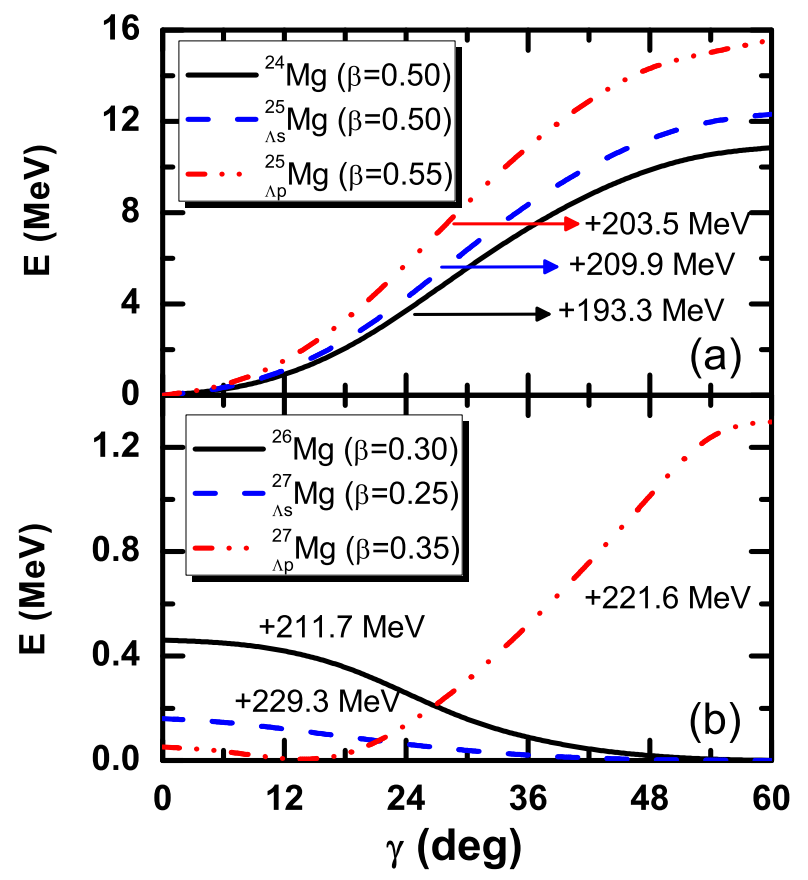

FIG. 6: (Color online) (a) The total energy of ${ }^{24} \mathrm{Mg},{ }_{\Lambda s}^{25} \mathrm{Mg}$ and ${ }_{\Lambda p}^{25} \mathrm{Mg}$ as a function of deformation parameter $\gamma$, where the deformation $\beta$ is fixed at the value of the global minimum of the energy surface. The energies are normalized to the energy of the global minimum. (b) Same as (a), but for ${ }^{26} \mathrm{Mg},{ }_{\Lambda s}^{27} \mathrm{Mg}$ and ${ }_{\Lambda p}^{27} \mathrm{Mg}$.

a function of $\beta$ and the different shape-driving effect of $\Lambda_{s}$ and $\Lambda_{p}$ shown in Fig. 4 . In other words, the $\Lambda_{s}$ drives the hypernucleus towards spherical shape, while the $\Lambda_{p}$ drives the hypernucleus towards large deformation, as has been pointed out in Ref. [45] based on the AMD calculations. For the axial asymmetric shapes, the energies of the three $p$-hyperon orbitals in ${ }_{\Lambda s}^{25} \mathrm{Mg}$ are apparently different from each other. The rotational bands with such configurations have been discussed recently based on the AMD model [74]. The energy of $\Lambda_{p}$ increases with $\gamma$, explaining the phenomenon that the $\Lambda_{p}$ drives hypernucleus towards prolate-like shape with slightly larger deformation $\beta$ (see Fig. 6 (b)).

\section{PROJECTED POTENTIAL ENERGY SURFACES FOR ${ }_{\Lambda}^{25} \mathrm{MG}$ WITH MICROSCOPIC PARTICLE-ROTOR MODEL}

The deformed mean-field states considered in the previous section break rotational symmetry and thus several angular momentum components are admixed in the wave functions. To compare with experimental data, one has to make a transformation from the intrinsic frame to the laboratory frame, which can be realized by introducing the technique of AMP. It can be implemented based on the mean-field wave function for the whole $\Lambda$ hypernuclei composed of an even-even nuclear core and one unpaired

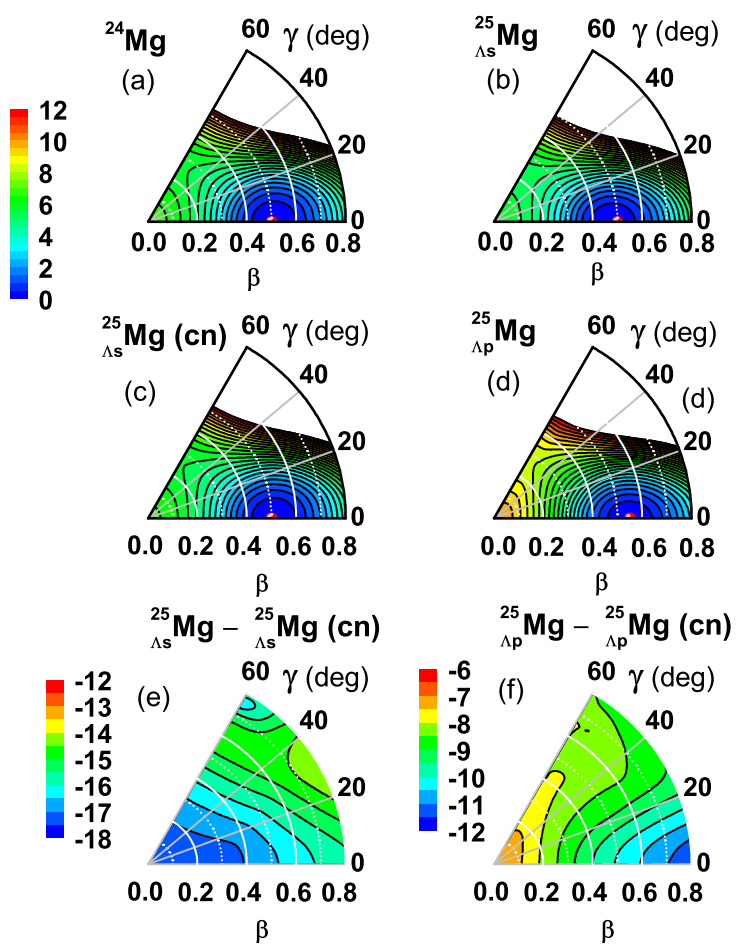

FIG. 7: (Color online) The potential energy surfaces of ${ }^{24} \mathrm{Mg}$ (a), ${ }_{\Lambda s}^{25} \mathrm{Mg}$ (b), ${ }_{\Lambda s}^{25} \mathrm{Mg}$ (cn) (c), ${ }_{\Lambda p}^{25} \mathrm{Mg}$ (d) in the $(\beta, \gamma)$ plane. The energies are normalized to the global minimum. The energy difference between ${ }_{\Lambda s}^{25} \mathrm{Mg}$ and its core nucleus (e), and that between ${ }_{\Lambda p}^{25} \mathrm{Mg}$ and its core nucleus (f) are also plotted. Two neighboring contour lines are separated by $0.5 \mathrm{MeV}$.
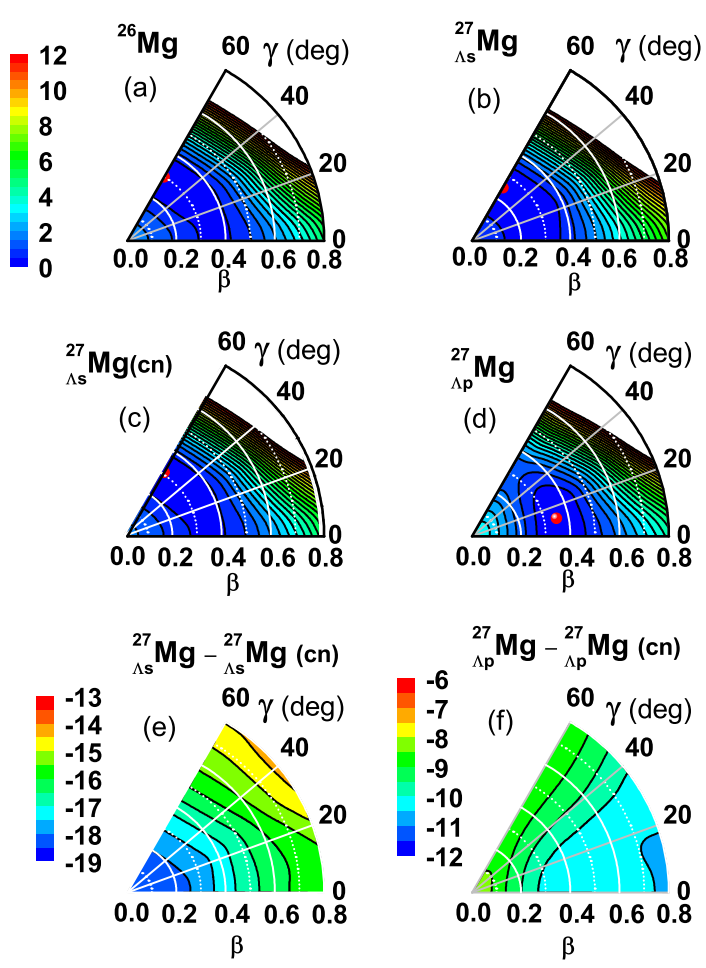

FIG. 8: (Color online) Same as Fig. 7, but for ${ }^{26} \mathrm{Mg},{ }_{\Lambda s}^{27} \mathrm{Mg}$, and ${ }_{\Lambda p}^{27} \mathrm{Mg}$. 

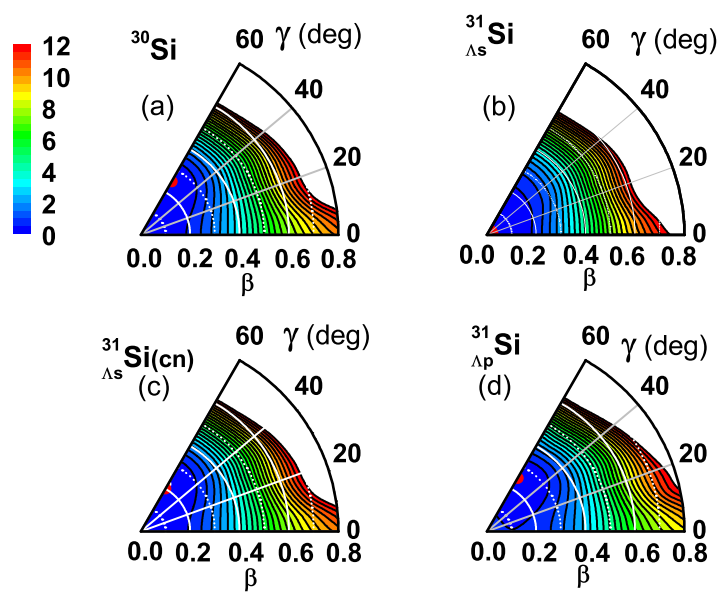

${ }_{\Lambda \mathrm{s}}^{31} \mathrm{Si}-{ }_{\Lambda \mathrm{s}}^{31} \mathrm{Si}(\mathrm{cn})$

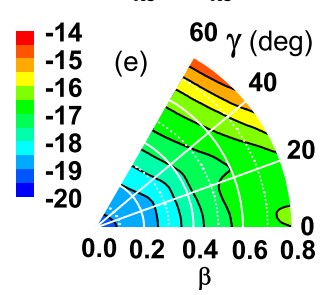

$$
{ }_{\Lambda \mathrm{p}}^{31} \mathrm{Si}-{ }_{\Lambda \mathrm{p}}^{31} \mathrm{Si}(\mathrm{cn})
$$

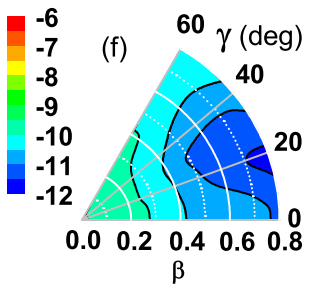

FIG. 9: (Color online) Same as Fig. 7, but for ${ }^{30} \mathrm{Si},{ }_{\Lambda_{s}}^{31} \mathrm{Si}$ and ${ }_{\Lambda p}^{31} \mathrm{Si}$.

$\Lambda$ particle. Rather than implementing the AMP for oddmass nuclear system, however, we instead apply the microscopic particle-rotor model (PRM) [13] to calculate the PESs for the hypernuclei.

The microscopic PRM was developed recently by the present authors using the transition densities from the multi-reference DFT calculation [75, 76]. In this model, the wave function of $\Lambda$ hypernucleus is constructed as

$$
\Psi_{I M}\left(\boldsymbol{r}_{\Lambda},\left\{\boldsymbol{r}_{N}\right\}\right)=\sum_{j \ell J} \mathscr{R}_{j \ell J}\left(r_{\Lambda}\right) \mathscr{F}_{j \ell J}^{I M}\left(\hat{\boldsymbol{r}}_{\Lambda},\left\{\boldsymbol{r}_{N}\right\}\right),
$$

where $\mathscr{F}_{j \ell J}^{I M}$ is given by

$$
\mathscr{F}_{j \ell J}^{I M}\left(\hat{\boldsymbol{r}}_{\Lambda},\left\{\boldsymbol{r}_{N}\right\}\right)=\left[\mathscr{Y}_{j \ell}\left(\hat{\boldsymbol{r}}_{\Lambda}\right) \otimes \Phi_{J}\left(\left\{\boldsymbol{r}_{N}\right\}\right)\right]^{(I M)}
$$

with $\boldsymbol{r}_{\Lambda}$ and $\boldsymbol{r}_{N}$ being the coordinates of the $\Lambda$ hyperon and the nucleons, respectively. Here, $I$ is the total angular momentum and $M$ is its projection onto the $z$-axis for the whole $\Lambda$ hypernucleus. $\mathscr{R}_{j \ell J}\left(r_{\Lambda}\right)$ and $\mathscr{Y}_{j \ell}\left(\hat{\boldsymbol{r}}_{\Lambda}\right)$ are the four-component radial wave function and the spinangular wave function for the $\Lambda$ hyperon, respectively. In this paper, the wave function $\Phi_{J}\left(\left\{\boldsymbol{r}_{N}\right\}\right)$ for the nuclear core is chosen as the projected mean-field wave function with different intrinsic deformation $\beta$, that is,

$$
\left|\Phi_{J M_{J}}(\beta)\right\rangle=\hat{P}_{M_{J} K}^{J} \hat{P}^{N} \hat{P}^{Z}|\varphi(\beta)\rangle,
$$

where $\hat{P}_{M_{J} K}^{J}$ is the projection operator onto a good number of angular momentum, while $\hat{P}^{N}$ and $\hat{P}^{Z}$ are those

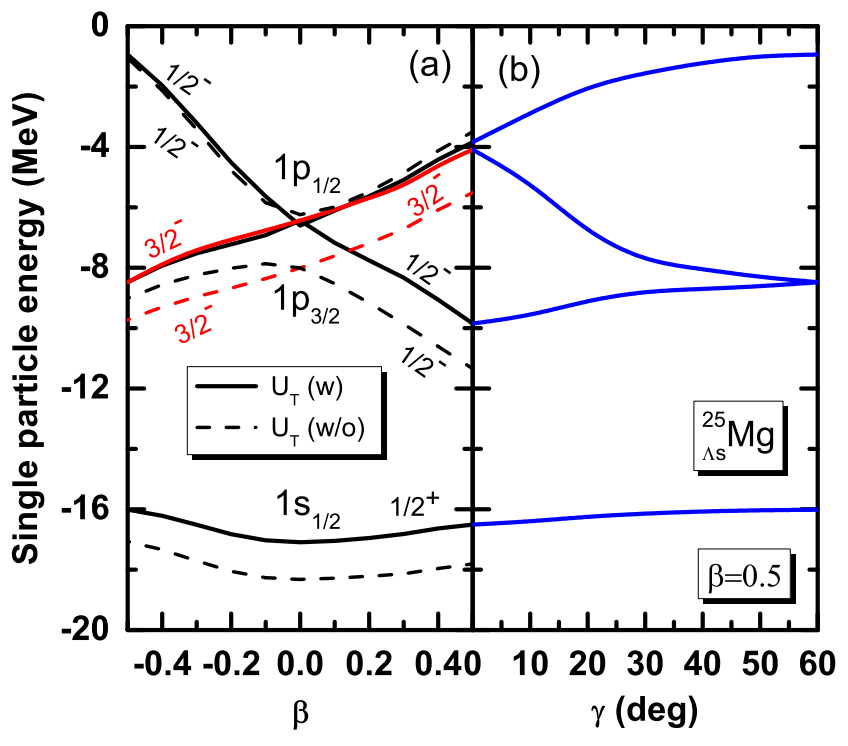

FIG. 10: (Color online) The single-particle energies of $\Lambda$ hyperon in ${ }_{\Lambda s}^{25} \mathrm{Mg}$ as a function of deformation parameters $\beta$ and $\gamma$ from the calculation with the PC-F1 and PCY-S1 for the $N N$ and $N \Lambda$ interactions, respectively. In the panel (a), the energy levels from the calculation without the tensor potential $U_{T}(11 \mathrm{~b})$ are also plotted with the dashed lines.

for neutron and proton numbers, respectively. The total energy of a $\Lambda$ hypernucleus with spin-parity $I^{\pi}$ corresponding to deformation $\beta$ of the core nucleus is defined as the energy $E_{I}$ of the lowest solution of the equation $\hat{H}\left|\Psi_{I M}\right\rangle=E_{I}\left|\Psi_{I M}\right\rangle$, from which one can derive the coupled-channels equations for $\mathscr{R}_{j \ell J}\left(r_{\Lambda}\right)$. More details on the microscopic PRM for $\Lambda$ hypernuclei are given in Refs. [13, 77].

Figure 11 shows the resultant PES $E_{I}(\beta)$ for ${ }_{\Lambda}^{25} \mathrm{Mg}$ with spin-parity of $I^{\pi}=1 / 2^{+}$and $1 / 2^{-}$as a function of the deformation $\beta$ of the core nucleus. The energy curve for the $3 / 2^{-}$state is almost the same as the that for the $1 / 2^{-}$state and is therefore not shown in the figure. In these calculations, only the leading-order fourfermion coupling terms (3) are taken into account for the $N \Lambda$ interaction with coupling strengths fitted to the $\Lambda$ binding energy from the coupled-channel PRM calculation [13] to the value $B_{\Lambda}=16.6 \mathrm{MeV}$ from the triaxial RMF calculation for ${ }_{\Lambda s}^{25} \mathrm{Mg}$. For comparison, the PES for the core nucleus ${ }^{24} \mathrm{Mg}$ with projection onto the particle numbers and angular momentum $J=0$ is also plotted. (The PESs for ${ }_{\Lambda}^{25} \mathrm{Mg}$ are calculated by coupling the hyperon to several $J$ states of the core nucleus built on the deformed mean-field state.) We note that the $1 / 2^{+}$state is dominated by the configuration with $\Lambda$ in $s$ orbital, while the $1 / 2^{-}$state is dominated by the configurations of $\left[p_{1 / 2} \otimes 0^{+}\right]$and $\left[p_{3 / 2} \otimes 2^{+}\right]$at nonzero deformation $\beta$.

The impurity effect of $\Lambda$ in the $s$ and $p$ orbitals on the PES after restoration of rotational symmetry can be inferred from the comparison of the PESs for ${ }_{\Lambda}^{25} \mathrm{Mg}$ with the projected PES (N\&Z, $J=0)$ for ${ }^{24} \mathrm{Mg}$. It is shown 


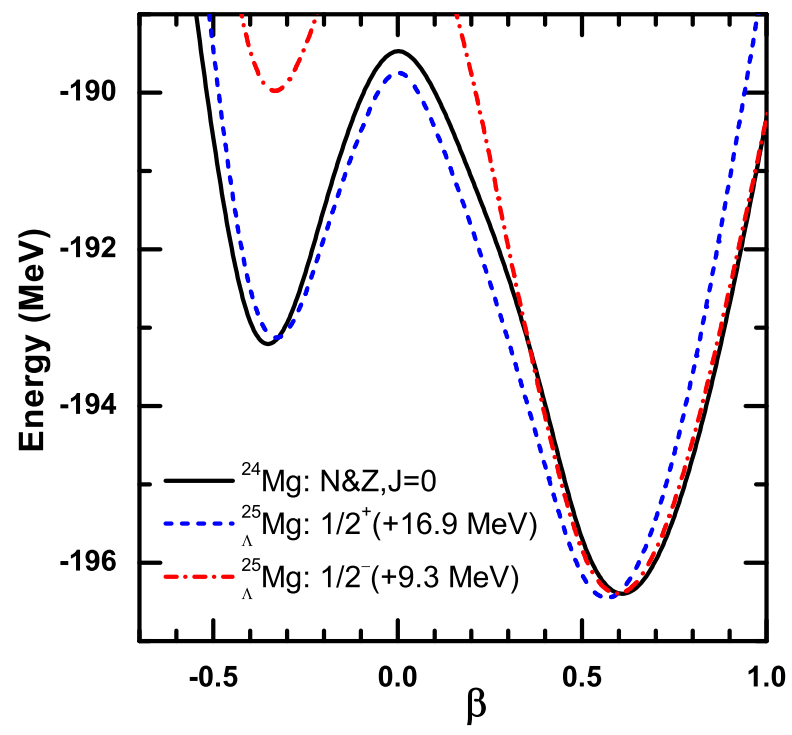

FIG. 11: (Color online) A comparison of the particle-number and angular-momentum projected PES (the solid curve) of ${ }^{24} \mathrm{Mg}$ with the PESs with spin-parity of $I^{\pi}=1 / 2^{+}$(the dashed curve) and $1 / 2^{-}$(the dash-dotted curve) for ${ }_{\Lambda}^{25} \mathrm{Mg}$ obtained with the microscopic particle-rotor model.

that the PES for ${ }_{\Lambda}^{25} \mathrm{Mg}$ with $I^{\pi}=1 / 2^{+}$has a global energy minimum at a slightly smaller deformation $\beta=0.55$ than the deformation $\beta=0.60$ for ${ }^{24} \mathrm{Mg}$. It confirms the conclusion drawn from the mean-field results shown in Fig. 4(a). For ${ }_{\Lambda}^{25} \mathrm{Mg}$ with $1 / 2^{-}$, the energies of the spherical and oblate deformed shapes with respect to the prolate minimum are significantly increased compared with those for the core nucleus with $J=0$. However, it is difficult to assess the change of the collectivity for this case, which depends on the distribution of the weight function in deformation plane. In order to determine such distribution, one could carry out the microscopic PRM calculation by coupling the $\Lambda$ to the configuration mixed nuclear core states, as has been done for ${ }_{\Lambda}^{9} \mathrm{Be}$ in Ref. [13]. However, this method is currently limited only to axial deformations. Alternatively, one can introduce a triaxial GCM or 5DCH method for the core nucleus to examine the $\Lambda$ impurity effect in a quantitative way. Since the former is very time-consuming for a systematic study, the $5 \mathrm{DCH}$ is adopted in the subsequent study.

\section{BEYOND MEAN-FIELD STUDY OF CORE NUCLEI WITH MICROSCOPIC COLLECTIVE HAMILTONIAN METHOD}

In the $5 \mathrm{DCH}$ approach, the collective excitations of the core nucleus are described with the following collective Hamiltonian,

$$
\hat{H}=\hat{T}_{\text {vib }}+\hat{T}_{\text {rot }}+V_{\text {coll }}
$$

where the first two terms are the vibrational kinetic energy

$$
\begin{aligned}
\hat{T}_{\mathrm{vib}}= & -\frac{\hbar^{2}}{2 \sqrt{w r}}\left\{\frac { 1 } { \beta ^ { 4 } } \left[\frac{\partial}{\partial \beta} \sqrt{\frac{r}{w}} \beta^{4} B_{\gamma \gamma} \frac{\partial}{\partial \beta}\right.\right. \\
& \left.-\frac{\partial}{\partial \beta} \sqrt{\frac{r}{w}} \beta^{3} B_{\beta \gamma} \frac{\partial}{\partial \gamma}\right]+\frac{1}{\beta \sin 3 \gamma}\left[-\frac{\partial}{\partial \gamma}\right. \\
& \left.\left.\sqrt{\frac{r}{w}} \sin 3 \gamma B_{\beta \gamma} \frac{\partial}{\partial \beta}+\frac{1}{\beta} \frac{\partial}{\partial \gamma} \sqrt{\frac{r}{w}} \sin 3 \gamma B_{\beta \beta} \frac{\partial}{\partial \gamma}\right]\right\},
\end{aligned}
$$

and the rotational kinetic energy

$$
\hat{T}_{\mathrm{rot}}=\frac{1}{2} \sum_{k=1}^{3} \frac{\hat{J}_{k}^{2}}{\mathcal{I}_{k}}
$$

with $\hat{J}_{k}$ denoting the components of the angular momentum in the body-fixed frame of a nucleus. Two quantities that appear in the vibrational kinetic energy, that is, $r=B_{1} B_{2} B_{3}$ and $w=B_{\beta \beta} B_{\gamma \gamma}-B_{\beta \gamma}^{2}$, determine the volume element in the collective space. The mass parameters $B_{\beta \beta}, B_{\beta \gamma}$ and $B_{\gamma \gamma}$, as well as the moments of inertia $\mathcal{I}_{k}$, depend on the quadrupole deformation variables $\beta$ and $\gamma$,

$$
\mathcal{I}_{k}=4 B_{k} \beta^{2} \sin ^{2}(\gamma-2 k \pi / 3), k=1,2,3,
$$

and are determined by the triaxial RMF $+\mathrm{BCS}$ calculations in cranking approximation [78].

The third term $V_{\text {coll }}$ in Eq. (21) is a collective potential given by

$$
V_{\text {coll }}(\beta, \gamma)=\tilde{E}_{\text {tot }}-\triangle V_{\mathrm{vib}}(\beta, \gamma)-\triangle V_{\mathrm{rot}}(\beta, \gamma),
$$

where $\triangle V_{\text {vib }}(\beta, \gamma)$ and $\Delta V_{\text {rot }}(\beta, \gamma)$ are the zero-pointenergy of vibrational and rotational motions, respectively. The $\tilde{E}_{\text {tot }}$ is given by the summation of total energy for the core nucleus inside the hypernucleus and the $N \Lambda$ interaction energy $\varepsilon_{N \Lambda}(\boldsymbol{r})(6 \mathrm{~b})$ which carries most of information on the $\Lambda$ impurity effect.

The eigenvalue problem with the collective Hamiltonian is solved by expanding eigenfunctions in terms of a complete set of basis functions that depend on the five degrees of freedom: the deformation variables $\beta$ and $\gamma$, and the Euler angles $\phi, \theta$ and $\psi$ [79].

Figure 12 shows the parameters in the $5 \mathrm{DCH}$ as a function of quadrupole deformation $\beta$ for ${ }^{24} \mathrm{Mg}$ and the core nucleus inside ${ }_{\Lambda s}^{25} \mathrm{Mg}$ and ${ }_{\Lambda p}^{25} \mathrm{Mg}$. These are the collective potential $V_{\text {coll }}$, the moment of inertia along $x$-direction $\mathcal{I}_{x}$, the rms radius of protons, and the mass parameters $B_{\beta \beta}$. It is shown that the collective potentials for the core nucleus inside ${ }_{\Lambda s}^{25} \mathrm{Mg}$ and ${ }_{\Lambda p}^{25} \mathrm{Mg}$ have a similar behavior as the projected PESs for ${ }_{\Lambda s}^{25} \mathrm{Mg}$ and ${ }_{\Lambda p}^{25} \mathrm{Mg}$ shown in Fig. 11. Moreover, the moment of inertia for the core nucleus around the energy minimum is significantly reduced by $\Lambda_{s}$ and $\Lambda_{p}$. The resultant energy spectrum is stretched as shown in Fig. 13 for the low-spin spectra of ground state band for the ${ }^{24} \mathrm{Mg}$ and the core nucleus inside ${ }_{\Lambda}^{25} \mathrm{Mg}$. The $\Lambda_{s}$ increases the excitation energy 


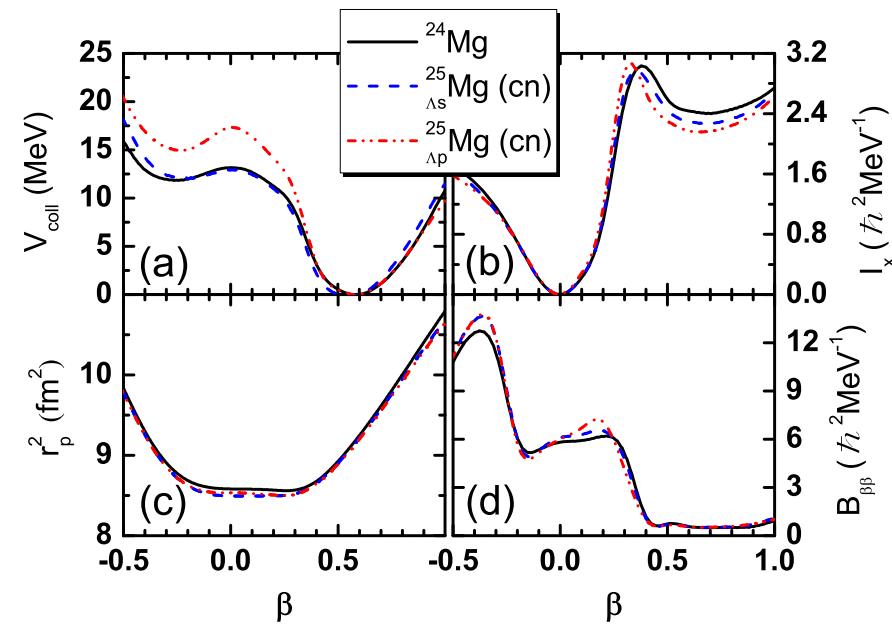

FIG. 12: (Color online) (a) The collective potential $V_{\text {coll }}$, (b) the moment of inertia along $x$-axis $\mathcal{I}_{x},(\mathrm{c})$ the mean squared radius of protons, and (d) the mass parameters $B_{\beta \beta}$ as a function of quadrupole deformation $\beta$ for ${ }^{24} \mathrm{Mg}$ and ${ }_{\Lambda}^{25} \mathrm{Mg}$ (cn) obtained with the fine-dimensional collective Hamiltonian $(5 \mathrm{DCH})$ approach.

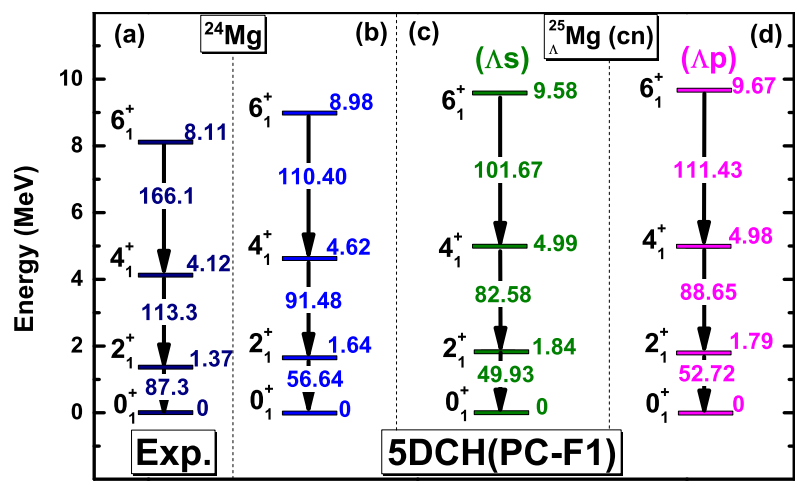

FIG. 13: (Color online) The low-spin spectra of the ground state band for ${ }^{24} \mathrm{Mg}$ (b) and the nuclear core of ${ }_{\Lambda}^{25} \mathrm{Mg}$ (c, d) obtained with the $5 \mathrm{DCH}$ method. The $B(E 2)$ values are in units of $\mathrm{e}^{2} \mathrm{fm}{ }^{4}$. The spectrum of ${ }^{24} \mathrm{Mg}$ is compared with the corresponding experimental data, taken from Ref. [80].

$E_{x}\left(2_{1}^{+}\right)$for the $2_{1}^{+}$state by $\sim 12.2 \%$ and reduces the $E 2$ transition strength $B\left(E 2: 2_{1}^{+} \rightarrow 0_{1}^{+}\right)$by $\sim 11.8 \%$, compared with the value of $\sim 7 \%$ and $\sim 9 \%$, respectively, found in our previous 5DCH calculation for the same nucleus based on the non-relativistic Skyrme EDFs [11, 81].

The sensitivity of $\Lambda$ impurity effect on nuclear collective properties to the underlying EDFs has been examined based on several sets of Skyrme EDFs with various pairing strengths for ${ }_{\Lambda s}^{45} \mathrm{~S}$ [81]. It has been found that although different Skyrme EDFs give somewhat different low-lying spectra for the core nucleus, they give similar and generally small size of $\Lambda$ impurity effect (typically within $5 \%$ ) on the spectroscopic observables. From this point of view, one can draw a conclusion that the present relativistic study yields the $\Lambda_{s}$ impurity effect on

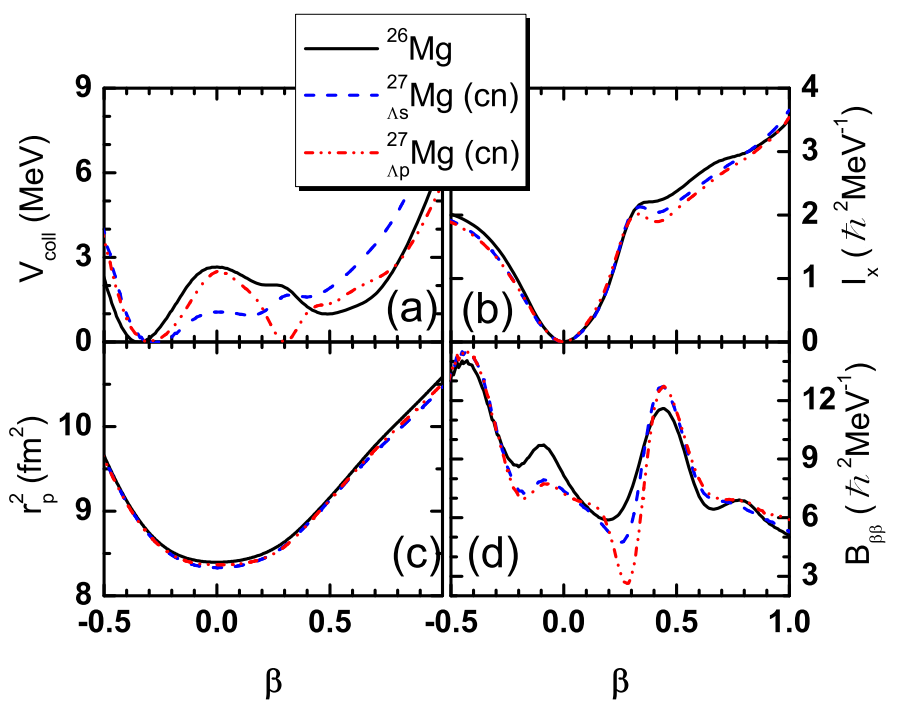

FIG. 14: (Color online) Same as Fig. 12, but for ${ }^{26} \mathrm{Mg}$ and nuclear core inside ${ }_{\Lambda}^{27} \mathrm{Mg}$.

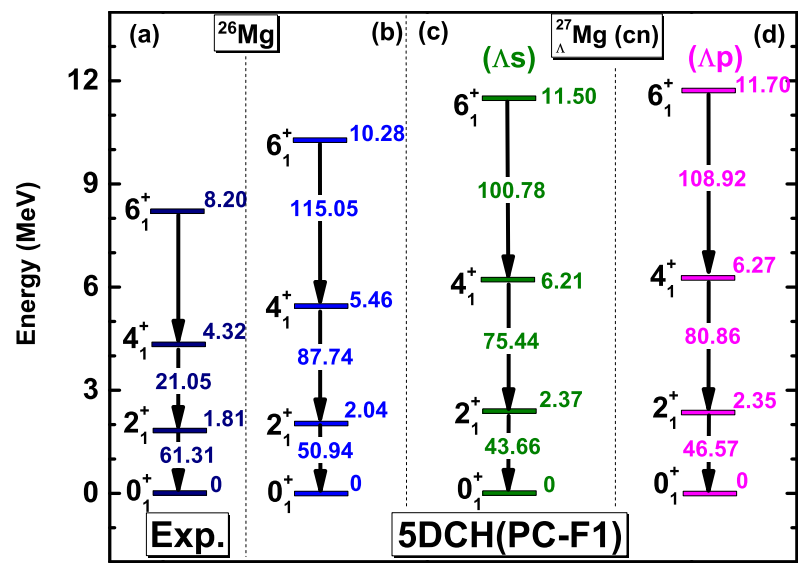

FIG. 15: (Color online) Same as Fig. 13, but for ${ }^{26} \mathrm{Mg}$ and ${ }_{\Lambda}^{27} \mathrm{Mg}(\mathrm{cn})$. The experimental data for ${ }^{26} \mathrm{Mg}$ are taken from Refs. [82, 83].

nuclear low-energy structure which is larger than that by non-relativistic Skyrme EDFs, similarly to the conclusion for mean-field calculations [44]. In addition, it is shown that the $\Lambda_{p}$ increases the excitation energy of $2_{1}^{+}$ state by $\sim 9.1 \%$ and reduces the $E 2$ transition strength $B\left(E 2: 2_{1}^{+} \rightarrow 0_{1}^{+}\right)$by $\sim 6.9 \%$. Moreover, we note that the excitation energy of $2_{2}^{+}$state is increased from 5.62 $\mathrm{MeV}$ to $5.88 \mathrm{MeV}$ and $6.67 \mathrm{MeV}$ due to the $\Lambda_{s}$ and $\Lambda_{p}$, respectively, which is consistent with the observation in Fig. 6.

Figure 14 shows the parameters in the $5 \mathrm{DCH}$ for ${ }^{26} \mathrm{Mg}$ and the core nuclei inside ${ }_{\Lambda s}^{27} \mathrm{Mg}$ and ${ }_{\Lambda p}^{26} \mathrm{Mg}$. Similar to the PESs in Fig. 8, the collective potential $V_{\text {coll }}$ is rather different for the ${ }_{\Lambda s}^{27} \mathrm{Mg},{ }_{\Lambda p}^{27} \mathrm{Mg}$ and the core nucleus ${ }^{26} \mathrm{Mg}$. Similarly to the ${ }^{24} \mathrm{Mg}$ case, the $\Lambda_{s}$ and $\Lambda_{p}$ apparently reduce the moments of inertia around the prolate minimum of the collective potential. The competition of 


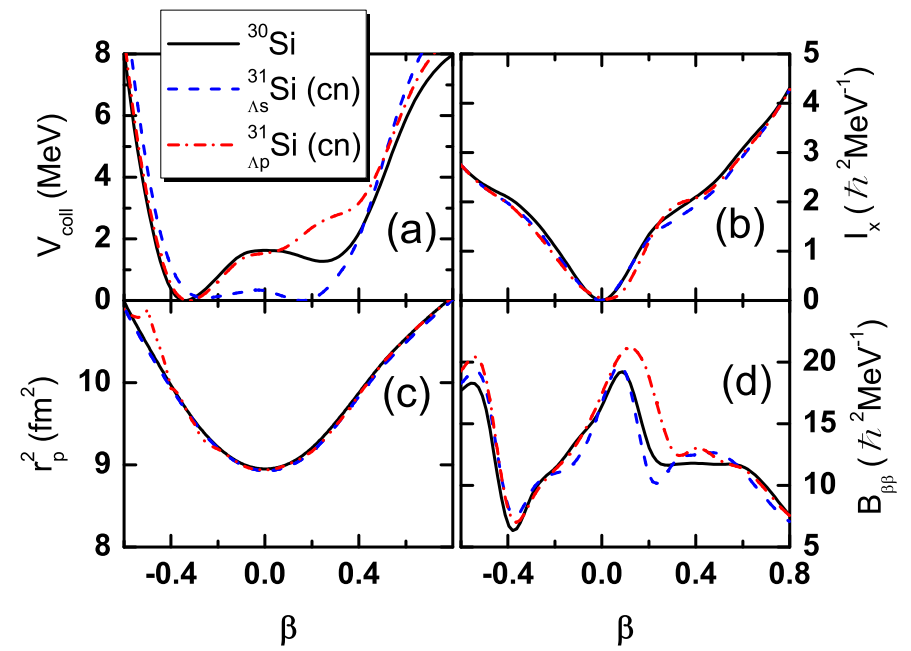

FIG. 16: (Color online) Same as Fig. 12, but for ${ }^{30} \mathrm{Si}$ and nuclear core inside ${ }_{\Lambda}^{31} \mathrm{Si}$.

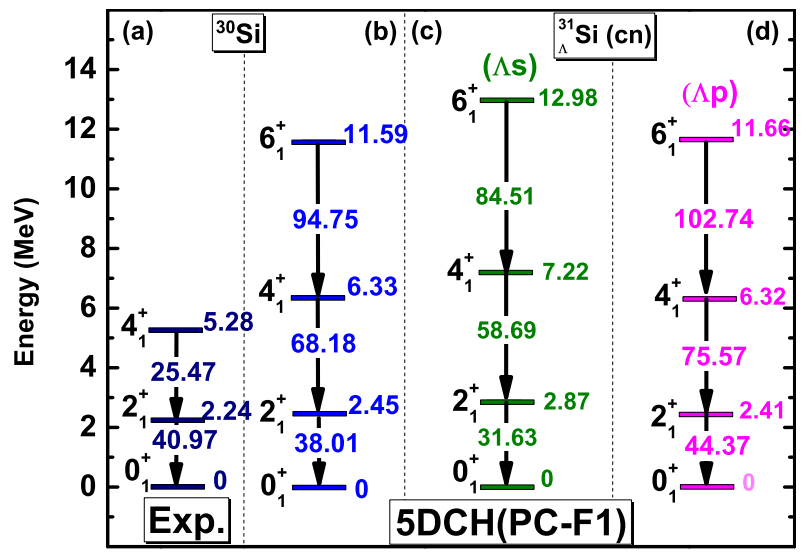

FIG. 17: (Color online) Same as Fig. 13, but for ${ }^{30} \mathrm{Si}$ and ${ }_{\Lambda}^{31} \mathrm{Si}(\mathrm{cn})$. The experimental data for ${ }^{30} \mathrm{Si}$ are taken from Refs. [82, 83].

the changes in the collective potential and the collective parameters results in the $\Lambda$ impurity effect on the lowenergy excitations. Figure 15 shows the low-spin spectra of the ground-state band for the ${ }^{26} \mathrm{Mg}$ and ${ }_{\Lambda}^{27} \mathrm{Mg}$. It is shown that the $\Lambda_{s}$ increases the excitation energy of the $2_{1}^{+}$state by $\sim 16.2 \%$ and reduces the $B\left(E 2: 2_{1}^{+} \rightarrow 0_{1}^{+}\right)$ by $\sim 14.3 \%$. Moreover, the $\Lambda_{p}$ increases the excitation energy of the $2_{1}^{+}$state by $\sim 15.2 \%$ and reduces the $B\left(E 2: 2_{1}^{+} \rightarrow 0_{1}^{+}\right)$by $\sim 8.6 \%$. The excitation energy of the $2_{2}^{+}$state is increased from $3.92 \mathrm{MeV}$ to $4.63 \mathrm{MeV}$ and $4.68 \mathrm{MeV}$ due to the $\Lambda_{s}$ and $\Lambda_{p}$, respectively.

Figure 16 shows the collective parameters for ${ }^{30} \mathrm{Si}$ and ${ }_{\Lambda}^{31} \mathrm{Si}$. In contrast to the $\Lambda_{p}$ effect in ${ }_{\Lambda s}^{25,27} \mathrm{Mg}$, the $\Lambda_{p}$ increases the mass parameters in most of the deformation regions. Together with the well-developed oblate minimum, the $\Lambda_{p}$ increases the nuclear collectivity as shown in Fig. 17. The $\Lambda_{p}$ increases significantly the $B\left(E 2: 2_{1}^{+} \rightarrow 0_{1}^{+}\right)$of the core nucleus ${ }^{30} \mathrm{Si}$ by $\sim 16.7 \%$ and decreases slightly the excitation energy of $2_{1}^{+}$state.

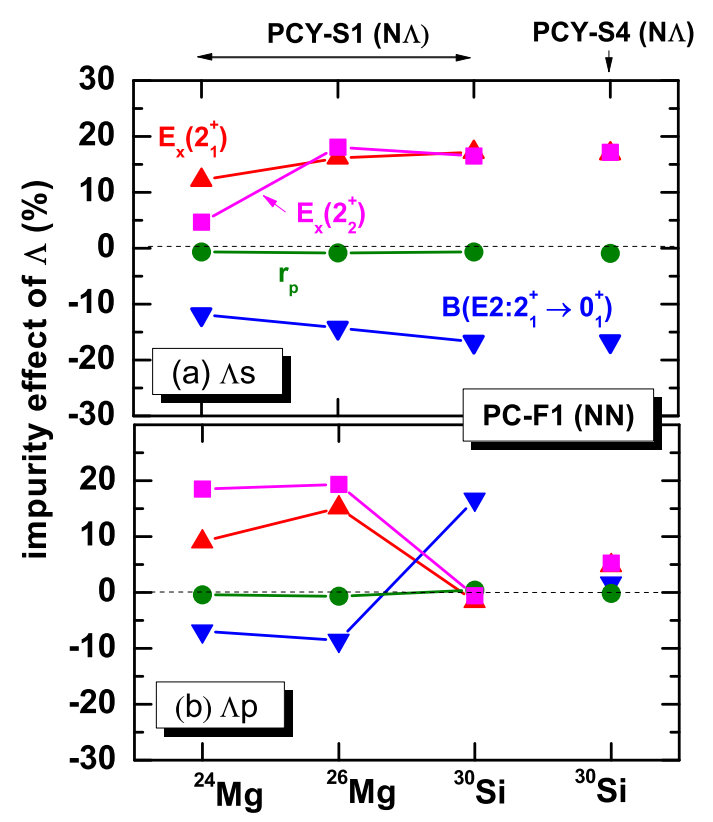

FIG. 18: (Color online) The impurity effect of $\Lambda_{s}$ and $\Lambda_{p}$ hyperon on the excitation energies $E_{x}\left(2_{1}^{+}\right)$and $E_{x}\left(2_{2}^{+}\right)$for the lowest two $2^{+}$states, the proton root-mean-squared radius $r_{p}$ of ground state $\left(0_{1}^{+}\right)$, and the $E 2$ transition strength $B(E 2$ : $2_{1}^{+} \rightarrow 0_{1}^{+}$) in ${ }^{24,26} \mathrm{Mg}$ and ${ }^{30} \mathrm{Si}$ from the $5 \mathrm{DCH}$ calculations based on the PC-F1 and PCY-S1 forces for the $N N$ and $N \Lambda$ interactions, respectively. For comparison, the impurity effect of $\Lambda_{s}$ and $\Lambda_{p}$ on ${ }^{30} \mathrm{Si}$ calculated with the $N \Lambda$ interaction of PCY-S4 [62] is also plotted in the last column.

The excitation energy of the $2_{2}^{+}$state is altered from 4.28 $\mathrm{MeV}$ to $4.99 \mathrm{MeV}$ and $4.26 \mathrm{MeV}$ due to the $\Lambda_{s}$ and $\Lambda_{p}$, respectively.

The impurity effect of $\Lambda_{s}$ and $\Lambda_{p}$ is summarized in Fig. 18. The change in the proton root-mean-squared radius of ground state is within $1 \%$ for all the three nuclei. It confirms that the reduction/enhancement of the $B(E 2)$ value for the core nucleus by adding a $\Lambda$ particle mainly originates from the modification of nuclear collective potential, the moment of inertia, and the mass parameters, rather than a shrinkage or an expansion of proton distribution as found in light nuclear systems with cluster structures [5]. One can also see that the changes in the excitation energies $E_{x}\left(2_{1}^{+}\right)$and $E_{x}\left(2_{2}^{+}\right)$for the lowest two $2^{+}$states are similar to one another. To examine the model-dependence of the $\Lambda$ impurity effect for ${ }^{30} \mathrm{Si}$, we also plot the results by the PCY-S4 $N \Lambda$ interaction in Fig. 18. It shows again that the impurity effect of $\Lambda_{s}$ is much less sensitive to the $N \Lambda$ interaction than that of $\Lambda_{p}$, that is consistent with the potential energy surfaces in Fig. 5. 


\section{SUMMARY}

We have established a triaxially deformed relativistic mean-field approach for $\Lambda$ hypernuclei based on a pointcoupling EDF. Using the 5DCH method based on this approach, we have quantitatively studied the impurity effect of $\Lambda_{s}$ and $\Lambda_{p}$ hyperon on the low-energy collective excitations of ${ }^{24} \mathrm{Mg},{ }^{26} \mathrm{Mg}$, and ${ }^{30} \mathrm{Si}$. Besides, the quadrupole deformation effect on the $\Lambda$ binding energies of hypernuclei has been studied. In particular, the PESs of three $s d$-shell $\Lambda$ hypernuclei ${ }^{25,27} \mathrm{Mg}$ and ${ }_{\Lambda}^{31} \mathrm{Si}$, as well as their core nucleus in $(\beta, \gamma)$ deformation plane has been calculated. The low-lying states of the core nuclei before and after adding $\Lambda$ hyperon in the lowest positive-parity $\left(\Lambda_{s}\right)$ and the negative-parity $\left(\Lambda_{p}\right)$ states have also been discussed. Moreover, the PESs of ${ }_{\Lambda}^{25} \mathrm{Mg}$ with spin-parity of $I^{\pi}=1 / 2^{+}$and $1 / 2^{-}$have been obtained with the microscopic PRM and compared with the PES of the core nucleus with $J^{\pi}=0^{+}$. Our main findings in the present studies are summarized as follows:

- The quadrupole deformation decreases the $\Lambda_{s}$ binding energy and increases the $\Lambda_{p}$ binding energy in $\Lambda$ hypernucleus.

- The potential energy surfaces of the whole $\Lambda$ hypernuclei could be significantly different from those of the core nuclei without the hyperon impurity. In general, the hypernuclei with a $\Lambda_{s}\left(\Lambda_{p}\right)$ have an energy minimum with smaller (or larger) deformation than the core nucleus. However, the potential energy surfaces of the core nuclei inside the hypernuclei are very similar to that of the nuclei without hyperon.
- Quantitatively, the $\Lambda_{s}$ increases the excitation energy of the $2_{1}^{+}$state and decreases the $E 2$ transition strength from this state to the ground state in the core nucleus by $12 \%-17 \%$, about twice larger than the value found in our previous $5 \mathrm{DCH}$ study based on the non-relativistic Skyrme EDFs. However, $\Lambda_{p}$ can either increase or decrease the collectivity of the core nucleus depending on the competition between the changes in the potential energy surface and the collective parameters.

Finally, we emphasize that the generalization of our triaxial RMF approach for hypernuclei to multi-strangeness systems is straightforward. Moreover, the present approach provides a starting point to carry out a beyond mean-field calculation of the low-lying states of $\Lambda$ hypernuclei by introducing the techniques of exact projections and GCM $[47,55]$ for the odd-mass system, the results of which can be compared with those of the microscopic PRM [13] based on the same relativistic point-coupling EDF.

\section{Acknowledgments}

We thank E. Hiyama, T. Koike, M. Isaka and S. G. Zhou for fruitful discussions. W. X. X. also thanks the Institute of Theoretical Physics in Chinese Academy of Sciences and the Nuclear Theory Group in Tohoku University for their hospitality during her visit. This work was supported by the NSFC under Grant Nos. 11475140, 11305134, 11105110, and 11105111, the Tohoku University Focused Research Project "Understanding the origins for matters in universe" and JSPS KAKENHI Grant Number 26400263.
[1] M. Danysz and J. Pniewski, Philos. Mag. 44, 348 (1953).

[2] M. Danysz and J. Pniewski, Bull. Acad. Pol. Sci. III 1, 42 (1953).

[3] N. Glendenning, Compact Stars, Springer-Verlag, New York (2000).

[4] T. Motoba, H. Bandō, and K. Ikeda, Prog. Theor. Phys. 70, 189 (1983).

[5] E. Hiyama, M. Kamimura, K. Miyazaki, and T. Motoba, Phys. Rev. C 59, 2351 (1999).

[6] K. Tanida, H. Tamura, D. Abe, H. Akikawa, K. Araki, H. Bhang, T. Endo, Y. Fujii, T. Fukuda, O. Hashimoto, K. Imai, H. Hotchi, Y. Kakiguchi, J. H. Kim, Y. D. Kim, T. Miyoshi, T. Murakami, T. Nagae, H. Noumi, H. Outa, K. Ozawa, T. Saito, J. Sasao, Y. Sato, S. Satoh, R. I. Sawafta, M. Sekimoto, T. Takahashi, L. Tang, H. H. Xia, S. H. Zhou, and L. H. Zhu, Phys. Rev. Lett. 86, 1982 (2001).

[7] E. Hiyama, M. Kamimura, T. Motoba, T. Yamada, and Y. Yamamoto, Phys. Rev. C 53, 2075 (1996).

[8] D. Vretenar, W. Pöschl, G. A. Lalazissis, and P. Ring, Phys. Rev. C 57, R1060 (1998).

[9] M. T. Win and K. Hagino,
Phys. Rev. C 78, 054311 (2008).

[10] B.-N. Lu, E.-G. Zhao, and S.-G. Zhou, Phys. Rev. C 84, 014328 (2011).

[11] J. M. Yao, Z. P. Li, K. Hagino, M. T. Win, Y. Zhang, and J. Meng, Nucl. Phys. A 868, 12 (2011).

[12] M. Isaka, H. Homma, M. Kimura, A. Doté, and A. Ohnishi, Phys. Rev. C 85, 034303 (2012).

[13] H. Mei, K. Hagino, J. M. Yao, and T. Motoba, Phys. Rev. C 90, 064302 (2014).

[14] K. Hagino, J. M. Yao, F. Minato, Z. P. Li, and M. T. Win, Nucl. Phys. A 914, 151 (2013).

[15] F. Minato, S. Chiba, and K. Hagino, Nucl. Phys. A 831, 150 (2009).

[16] F. Minato and S. Chiba, Nucl. Phys. A 856, 55 (2011).

[17] F. Hofmann, C. M. Keil, and H. Lenske, Phys. Rev. C 64, 025804 (2001).

[18] D. Davis, Nucl. Phys. A 754, 3 (2005), proceedings of the Eighth International Conference on Hypernuclear and Strange Particle Physics.

[19] R. Dalitz, Nucl. Phys. A 754, 14 (2005), proceedings of the Eighth International Conference on Hypernuclear and Strange Particle Physics. 
[20] O. Hashimoto and
Prog. Part. Nucl. Phys. 57, 564
[21] E.
E. Hiyama Prog. Part. Nucl. Phys. 63, 339 (2009).

[22] H.-J. Schulze, Nucl. Phys. A 835, 19 (2010).

[23] E. Hiyama, Few-Body Systems 53, 189 (2012).

[24] E. Botta, T. Bressani, and G. Garbarino, Euro. Phys. J. A 48 (2012).

[25] K. Hagino and J. M. Yao, arXiv:1410.7531v1 [nucl-th] (2014).

[26] H. Tamura, K. Tanida, D. Abe, H. Akikawa, K. Araki, H. Bhang, T. Endo, Y. Fujii, T. Fukuda, O. Hashimoto, K. Imai, H. Hotchi, Y. Kakiguchi, J. H. Kim, Y. D. Kim, T. Miyoshi, T. Murakami, T. Nagae, H. Noumi, H. Outa, K. Ozawa, T. Saito, J. Sasao, Y. Sato, S. Satoh, R. I. Sawafta, M. Sekimoto, T. Takahashi, L. Tang, H. H. Xia, S. H. Zhou, and L. H. Zhu, Phys. Rev. Lett. 84, 5963 (2000).

[27] M. Rayet, Ann. of Phys. (N.Y.) 102, 226 (1976).

[28] R. Brockmann and W. Weise, Phys. Lett. B 69, 167 (1977).

[29] J. Boguta and S. Bohrmann, Phys. Lett. 102B, 93 (1981).

[30] Y. Yamamoto, H. Bando, and J. Zofka, Prog. Theo. Phys. 80, 757 (1988).

[31] J. Mares and J. Zofka, Z. Phys. A 333, 209 (1989).

[32] M. Rufa, J. Schaffner, J. Maruhn, H. Stöcker, W. Greiner, and P.-G. Reinhard, Phys. Rev. C 42, 2469 (1990).

[33] Y. Sugahara and Prog. Theo. Phys. 92, 803 (1994).

H. Toki,

[34] J. Mareš and B. K. Jennings, Phys. Rev. C 49, 2472 (1994).

[35] C. M. Keil, F. Hofmann, and H. Lenske, Phys. Rev. C 61, 064309 (2000).

[36] H. F. Lu and J. Meng, Chin. Phys. Lett. 19, 1775 (2002).

[37] P. Finelli, N. Kaiser, D. Vretenar, and W. Weise, Nucl. Phys. A 831, 163 (2009).

[38] C. Y. Song, J. M. Yao, H. F. Lu, and J. Meng, Int. J. Mod. Phys. E 19, 2538 (2010).

[39] X.-R. Zhou, H.-J. Schulze, H. Sagawa, C.-X. Wu, and E.-G. Zhao, Phys. Rev. C 76, 034312 (2007).

[40] M. T. Win, K. Hagino, and T. Koike, Phys. Rev. C 83, 014301 (2011).

[41] A. Li, E. Hiyama, X.-R. Zhou, and H. Sagawa, Phys. Rev. C 87, 014333 (2013).

[42] B.-N. Lu, E. Hiyama, H. Sagawa, and S.-G. Zhou, Phys. Rev. C 89, 044307 (2014).

[43] R. $\mathrm{Xu}, \mathrm{C}$. $\mathrm{Wu}$, and Z. Ren, Nucl. Phys. A 933, 82 (2015).

[44] H.-J. Schulze, M. T. Win, K. Hagino, and H. Sagawa, Prog. Theor. Phys. 123, 569 (2010).

[45] M. Isaka, M. Kimura, A. Dote, and A. Ohnishi, Phys. Rev. C 83, 044323 (2011).

[46] J. M. Yao, J. Meng, P. Ring, and D. P. Arteaga, Phys. Rev. C 79, 044312 (2009).

[47] J. M. Yao, J. Meng, P. Ring, and D. Vretenar, Phys. Rev. C 81, 044311 (2010).

[48] J. M. Yao, H. Mei, H. Chen, J. Meng, P. Ring, and D. Vretenar, Phys. Rev. C 83, 014308 (2011).

[49] J. M. Yao, J. Meng, P. Ring, and D. P. Arteaga, Phys. Rev. C 79, 044312 (2009).

[50] Z. P. Li, T. Nikšić, D. Vretenar, J. Meng, G. A. Lalazissis, and P. Ring, Phys. Rev. C 79, 054301 (2009).

[51] Z. P. Li, T. Nikšić, D. Vretenar, P. Ring, and J. Meng, Phys. Rev. C 81, 064321 (2010).

[52] Z. P. Li, J. M. Yao, D. Vretenar, T. Nikšić, H. Chen, and J. Meng, Phys. Rev. C 84, 054304 (2011).

[53] H. Mei, J. Xiang, J. M. Yao, Z. P. Li, and J. Meng, Phys. Rev. C 85, 034321 (2012).

[54] Y. Fu, H. Mei, J. Xiang, Z. P. Li, J. M. Yao, and J. Meng, Phys. Rev. C 87, 054305 (2013).

[55] J. M. Yao, K. Hagino, Z. P. Li, J. Meng, and P. Ring, Phys. Rev. C 89, 054306 (2014).

[56] H. Y. Sang, X. S. Wang, H. F. Lü, J. M. Yao, and H. Sagawa, Phys. Rev. C 88, 064304 (2013).

[57] J. M. Yao, H. Chen, and J. Meng, Phys. Rev. C 74, 024307 (2006).

[58] L. S. Song, J. M. Yao, P. Ring, and J. Meng, Phys. Rev. C 90, 054309 (2014).

[59] J. M. Yao, L. S. Song, K. Hagino, P. Ring, and J. Meng, arXiv:1410.6326 [nucl-th] (2014) .

[60] T. Bürvenich, D. G. Madland, J. A. Maruhn, and P.-G. Reinhard, Phys. Rev. C 65, 044308 (2002).

[61] P. W. Zhao, Z. P. Li, J. M. Yao, and J. Meng, Phys. Rev. C 82, 054319 (2010).

[62] Y. Tanimura and K. Hagino, Phys. Rev. C 85, 014306 (2012).

[63] J. V. Noble, Phys. Lett. B 89, 325 (1980).

[64] A. Boussy, Phys. Lett. B 99, 305 (1981).

[65] J. K. Zhang and X. J. Qiu, Phys. Lett. B 152, 153 (1985).

[66] B. K. Jennings, Phys. Lett. B 246, 325 (1990).

[67] J. M. Yao, H. F. Lu, G. Hillhouse, and J. Meng, Chin. Phys. Lett. 25, 1629 (2008).

[68] M. Bender, K. Rutz, P.-G. Reinhard, and J. Maruhn, Euro. Phys. J. A 8, 59 (2000).

[69] J. Li, Y. Zhang, J. M. Yao, and J. Meng, Sci. China Phys. Mech. Astron. 52, 1586 (2009).

[70] H. D. Xu, Y. Wang, J. Li, and J. B. Lu, Nucl. Phys. A 929, 191 (2014).

[71] H. Hotchi, T. Nagae, H. Outa, H. Noumi, M. Sekimoto, T. Fukuda, H. Bhang, Y. Kim, J. Kim, H. Park, K. Tanida, O. Hashimoto, H. Tamura, T. Takahashi, Y. Sato, T. Endo, S. Satoh, J. Nishida, T. Miyoshi, T. Saitoh, T. Kishimoto, A. Sakaguchi, S. Ajimura, Y. Shimizu, T. Mori, S. Minami, M. Sumihama, R. Sawafta, and L. Tang, Phys. Rev. C 64, 044302 (2001).

[72] Q. N. Usmani and A. R. Bodmer, Phys. Rev. C 60, 055215 (1999).

[73] T. Motoba, Nucl. Phys. A 639, 135c (1998).

[74] M. Isaka, M. Kimura, A. Doté, and A. Ohnishi, Phys. Rev. C 87, 021304 (2013).

[75] J. M. Yao, H. Mei, and Z. P. Li, Phys. Lett. B 723, 459 (2013).

[76] J. M. Yao, M. Bender, and P.-H. Heenen, Phys. Rev. C 91, 024301 (2015).

[77] H. Mei, K. Hagino, J. M. Yao, and T. Motoba, in preparation (2014).

[78] T. Nikšić, Z. P. Li, D. Vretenar, L. Próchniak, J. Meng, and P. Ring, Phys. Rev. C 79, 034303 (2009).

[79] L. Pröchniak, K. Zajac, K. Pomorski, S. Rohozinski, and J. Srebrny, Nucl. Phys. A 648, 181 (1999).

[80] P. Endt, C. Alderliesten, F. Zijderhand, A. Wolters, and A. V. Hees, Nucl. Phys. A 510, 209 (1990).

[81] H. Mei, Z. P. Li, J. M. Yao, and K. Hagino, 
Int. J. Mod. Phys. E 21, 1250024 (2012).

[82] J. Tauren and R. B. Firest, Evaluated Nuclear Structure Data File http://ie.lbl.gov/TOI2003/index.asp .
(ENSDF),
Nuclear http://www.nndc.bnl.gov/.
Center

(NNDC), 